\title{
BMJ Open Evaluating the use of telemedicine in gynaecological practice: a systematic review
}

\author{
Sughashini Murugesu (D) ,,2 Nicolas Galazis, ${ }^{2,3}$ Benjamin P Jones (D) ,2,4 \\ Maxine Chan, ${ }^{2,4}$ Timothy Bracewell-Milnes, ${ }^{5}$ Yousra Ahmed-Salim, ${ }^{2}$ \\ Karen Grewal, ${ }^{2,4}$ Dirk Timmerman, ${ }^{2,6}$ Joseph Yazbek, ${ }^{2}$ Tom Bourne, 2,4,6 \\ Srdjan $\mathrm{Saso}^{2,4}$
}

To cite: Murugesu S, Galazis N, Jones BP, et al. Evaluating the use of telemedicine in gynaecological practice: a systematic review. BMJ Open 2020;10:e039457. doi:10.1136/ bmjopen-2020-039457

- Prepublication history for this paper is available online. To view these files, please visit the journal online (http://dx.doi. org/10.1136/bmjopen-2020039457).

Received 16 April 2020 Revised 23 October 2020 Accepted 30 October 2020

Check for updates

(c) Author(s) (or their employer(s)) 2020. Re-use permitted under CC BY-NC. No commercial re-use. See rights and permissions. Published by BMJ.

${ }^{1}$ Obstetrics and Gynaecology, Hillingdon Hospital NHS Trust, Uxbridge, UK

${ }^{2}$ Queen Charlotte's Hospital, Imperial College Healthcare NHS Trust, London, UK

${ }^{3}$ Obstetrics and Gynaecology, Northwick Park Hospital, Harrow, UK

${ }^{4}$ Institute for Reproductive Development and Biology, Imperial College London, London, UK

${ }^{5}$ Obstetrics and Gynaecology, Chelsea and Westminster NHS Hospital, London, UK

${ }^{6}$ Obstetrics and Gynaecology, University Hospitals KU Leuven, Leuven, Belgium

Correspondence to Dr Sughashini Murugesu; sugha.murugesu@gmail.com

\section{ABSTRACT}

Objectives The aim of this systematic review is to examine the use of telemedicine in the delivery and teaching of gynaecological clinical practice. To our knowledge, no other systematic review has assessed this broad topic.

Design Systematic review of all studies investigating the use of telemedicine in the provision of gynaecological care and education. The search for eligible studies followed the Preferred Reporting Items for Systematic Reviews and Meta-Analyses guidelines and focused on three online databases: PubMed, Science Direct and SciFinder.

Eligibility criteria Only studies within gynaecology were considered for this review. Studies covering only obstetrics and with minimal information on gynaecology, or clinical medicine in general were excluded. All English language, peer-reviewed human studies were included. Relevant studies published up to the date of final submission of this review were considered with no restrictions to the publication year.

Data extractions and synthesis Data extracted included author details, year of publication and country of the study, study aim, sample size, methodology, sample characteristics, outcome measures and a summary of findings. Data extraction and qualitative assessment were performed by the first author and crossed checked by the second author. Quality assessment for each study was assessed using the Newcastle-0ttawa scale.

Results A literature search carried out in August 2020 yielded 313 records published between 1992 and 2018. Following a rigorous selection process, only 39 studies were included for this review published between 2000 and 2018. Of these, 19 assessed gynaecological clinical practice, eight assessed gynaecological education, one both, and 11 investigated the feasibility of telemedicine within gynaecological practice. 19 studies were classified as good, 12 fair and eight poor using the Newcastle-0ttawa scale. Telecolposcopy and abortion care were two areas where telemedicine was found to be effective in potentially speeding up diagnosis as well as providing patients with a wide range of management options. Studies focusing on education demonstrated that telementoring could improve teaching in a range of scenarios such as live surgery and international teleconferencing.

\section{Strengths and limitations of this study}

- The review addresses the delivery of gynaecological clinical care and education remotely (telemedicine), a topic of immense importance as we are undergoing a period of global pandemic.

- To our knowledge, no other systematic review has assessed this broad topic.

- Large proportion of studies were observational in nature, without clear quantitative outcomes for statistical analysis.

Conclusions The results of this review are promising and demonstrate that telemedicine has a role to play in improving clinical effectiveness and education within gynaecology. Its applications have been shown to be safe and effective in providing remote care and training. In the future, randomised controlled studies involving larger numbers of patients and operators with measurable outcomes are required in order to be able to draw reliable conclusions.

\section{INTRODUCTION}

Moving into the 21st century, the exponential development of technology is driving change in the teaching and practice of gynaecology. ${ }^{1}$ The potential to reach a wider audience via targeted cost-effective innovation is almost inevitable, with an avenue opening up to improve access to healthcare and patient outcomes. ${ }^{2}$ Telemedicine (TM) can be defined as 'the use of medical information exchanged from one site to another via electronic communications to improve a patient's clinical health status'. ${ }^{3}$ It can be used for clinical care directly and as a method of educating trainees.

Within gynaecology, advocates of TM stress its potential role to aid diagnosis, treatment, follow-up and long-term care. Inadequate access to overall medical care has been reported as being related to a range of factors including 
poverty, the requirement of health insurance, geographic isolation from healthcare providers and lack of education. ${ }^{4-6}$ Difficult access to healthcare providers is not just an issue facing low-income countries. With the centralisation of tertiary services, which has shown to improve patient outcomes, access and availability can also be an issue in patients living in rural areas of high-income countries, as well as certain patients living in urban areas with mobility issues. ${ }^{7}$

Advocates of TM believe in the potential of providing more efficient care when compared with traditional means of care provision. This could be achieved by relatively cheap means using devices and programmes that are easily accessible, by minimising travel costs and reaching large numbers of patients. The need for effective and safe patient care through TM is ever more urgent in the current climax with the pandemic of COVID-19 necessitating delivery of care without exposing the patients and care providers. Indeed, during the pandemic, virtual gynaecology clinics have been taking place and there may be a place for such clinics to continue in the future for selected patients.

The aim of this systematic review is to assess the current evidence for the application of TM within gynaecology in terms of its impact and value on clinical care, education and feasibility. No such review has been performed in the literature to date.

\section{METHODS}

\section{Search strategy}

The systematic search followed Preferred Reporting Items for Systematic Reviews andMeta-Analyses guidelines. ${ }^{8}$ A bibliographic search of English language publications in three computerised databases (PubMed, Science Direct and SciFinder) was conducted. A bibliographic search of English language publications in the computerised database PubMed was conducted. PubMed was our primary database where controlled vocabulary (Medical Subject Headings (MeSH) words) was used separately and in combination. ${ }^{9}$ Free text words were also used on PubMed and on the supplementary databases: Scifinder and Science Direct.

The search terms, MeSH words and combinations of searches used are listed in table 1. Various combinations of "gynaecology" or "gynecology" with other relevant MeSH words such as "telementoring", "telemedicine", "teleconferencing", "mobile health", "telehealth", "ehealth" and "mhealth" were used for the literature search. Finally, the search was augmented by a snowball strategy, examining the references cited in primary sources and review manuscripts. The screening and selection process of the relevant studies conducted are shown on the figure 1.

\section{Study selection}

Only studies within gynaecology were considered for this review. Studies covering only obstetrics and with minimal information on gynaecology, or clinical medicine in general were excluded. All English language,
Table 1 Search and selection strategy

\begin{tabular}{ll}
$\begin{array}{l}\text { Database } \\
\text { searched }\end{array}$ & PubMed, Scifinder and Science Direct \\
\hline $\begin{array}{l}\text { Search } \\
\text { keywords }\end{array}$ & [gynaecology*] AND [telementoring] \\
& [gynaecology] AND [telemedicine] \\
& [gynaecology] AND [teleconferencing] \\
& [gynaecology] AND [telemonitoring] \\
& [gynaecology] AND [telepresence] \\
& [gynaecology] AND [interoperability] \\
& [gynaecology] AND [teleconsultation] \\
& [gynaecology] AND [remote care] \\
& [gynaecology] AND [mobile health] \\
& [gynaecology] AND [telehealth] \\
& [gynaecology] AND [ehealth] \\
& [gynaecology] AND [mhealth] \\
& [abortion care] AND [All above] \\
& [colposcopy] AND [All above]
\end{tabular}

Other sources Additional studies were identified through references of included studies and reviews

\begin{tabular}{|c|c|}
\hline $\begin{array}{l}\text { Inclusion } \\
\text { criteria }\end{array}$ & $\begin{array}{l}\text { 1. Published in English in peer-reviewed } \\
\text { journals } \\
\text { 2. Studies focusing on gynaecology only } \\
\text { 3. Studies investigating human clinical } \\
\text { practice }\end{array}$ \\
\hline $\begin{array}{l}\text { Exclusion } \\
\text { criteria }\end{array}$ & $\begin{array}{l}\text { 1. Papers not in English } \\
\text { 2. Full article not available } \\
\text { 3. Studies investigating animals }\end{array}$ \\
\hline $\begin{array}{l}\text { Categories of } \\
\text { studies }\end{array}$ & $\begin{array}{l}\text { 1. Gynaecology telemedicine studies } \\
\text { focused on education } \\
\text { 2. Gynaecology telemedicine studies } \\
\text { focused on clinical practice } \\
\text { 3. Gynaecology telemedicine studies } \\
\text { focused on education and clinical } \\
\text { practice } \\
\text { 4. Gynaecology telemedicine studies } \\
\text { focused on feasibility }\end{array}$ \\
\hline
\end{tabular}

"All the above combinations which contained "gynaecology" were also repeated with the word "gynecology".

peer-reviewed human studies were included. Relevant studies published up to August 2020 were considered with no restrictions to the publication year.

\section{Study screening}

The initial search for the relevant studies was performed by the first author (SM) and was independently repeated by the second author (NG). An overview of the search results and screening process is summarised in the study flow diagram (figure 1). The screening process was crosschecked by a senior author (SS). Disagreement between the reviewers was resolved by discussion until consensus was reached.

\section{Data extraction and analysis}

A data extraction spreadsheet was developed and agreed between the authors. The selected studies were 
Search results on Pubmed, Science Direct and SciFinder:

Search results: telementoring + gynaecology $=2$ Search results: telemedicine+gynaecology $=222$

Search results: teleconferencing + gynaecology $=4$

Search results: telemonitoring + gynaecology $=9$

Search results: telepresence+gynaecology $=7$

Search results: interoperability + gynaecology $=8$

Search results: teleconsultation + gynaecology $=61$

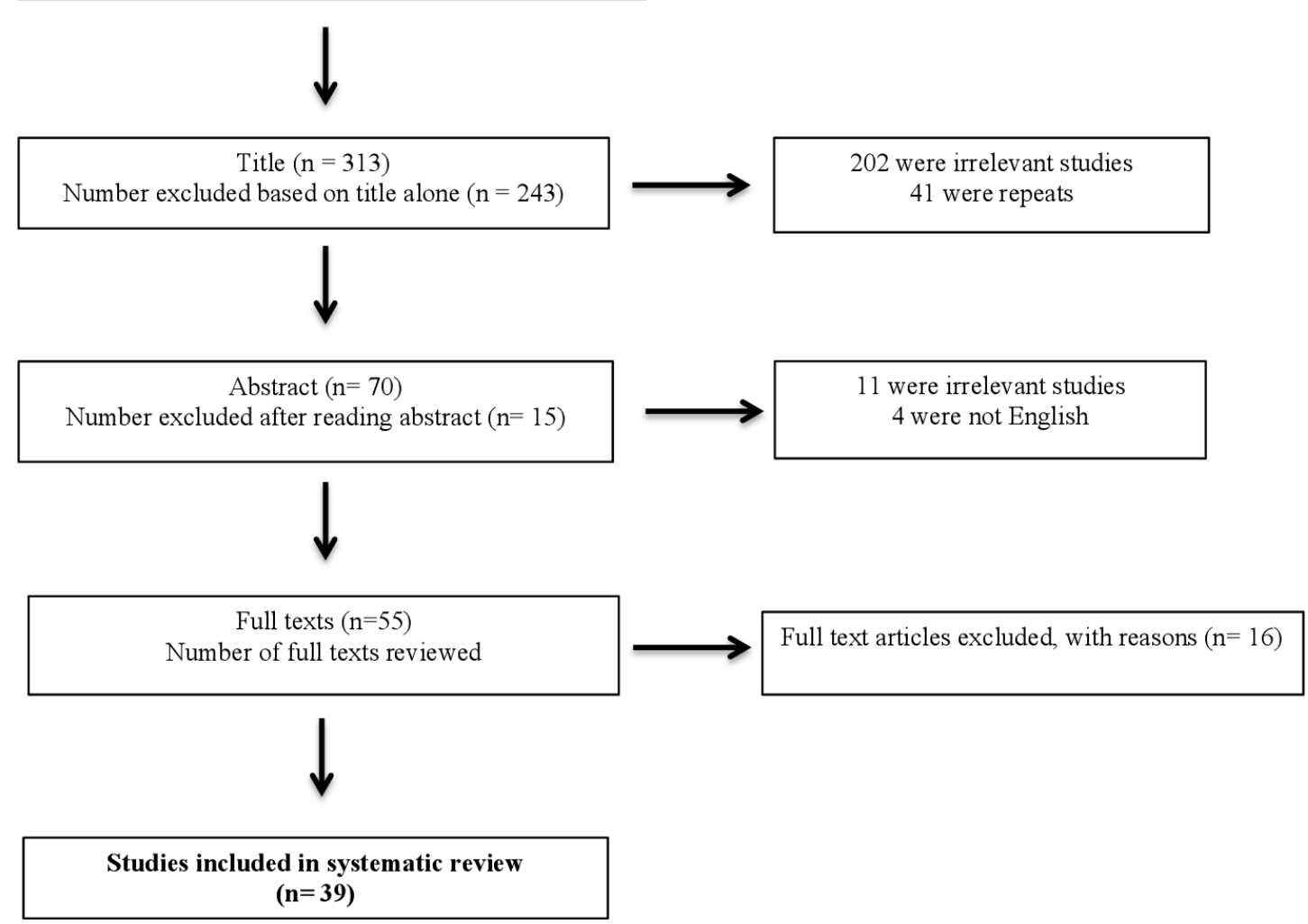

Figure 1 Preferred Reporting Items for Systematic Reviews andMeta-Analyses information flow chart.

comprehensively examined. Relevant data were extracted for each paper and inputted to the spreadsheet by the first author (SM) and subsequently crosschecked by the second author (NG). Data were then analysed qualitatively and summarised in the Results section. Because of the heterogeneity of the studies describing different modes of TM on different clinical topics within gynaecology, it was not possible to pool data together and perform a meta-analysis. The authors of the selected studies were not contacted to provide any information other than what was presented in the studies.

Quality assessment for each study was assessed using the Newcastle-Ottawa scale proforma. ${ }^{10}$ Using this quality assessment tool, each study is judged on eight items, categorised into three groups: the selection of the study groups; the comparability of the groups; and the ascertainment of either the exposure or outcome of interest for case-control or cohort studies, respectively. ${ }^{10}$ Disagreement regarding extracted data were resolved by discussion and deliberated on by a more senior author (SS).
Patient and public involvement

Patients were not involved in the design or required for recruitment in this systematic review.

\section{RESULTS}

The results are presented in the following tables: table 2 lists the topic and aim of the study, results and conclusions; table 3 contains descriptive factors related to technology such as the distance between the subjects and the tele-healthcare provider, the bandwidth and the device/ technology used; table 4 consists of more study characteristics including the duration of the study, the subjects (eg, cadavers, simulation, etc) and number of operators/ clinicians. The studies have also divided into four broad categories according to their topic: clinical, education, clinical and education and feasibility.

\section{Selected studies}

After the initial search though PubMed, a total of 313 records were screened. The publications dated from 


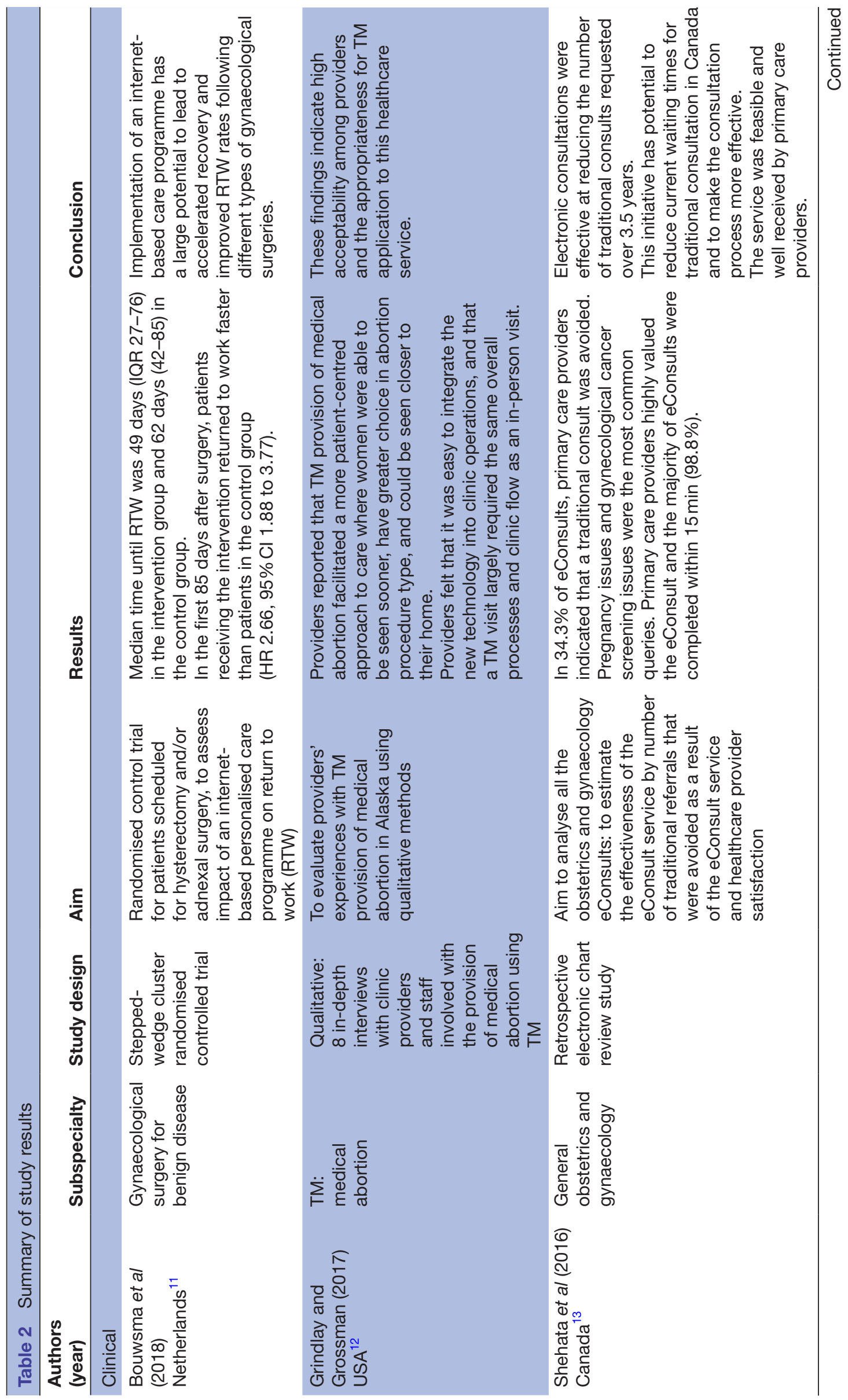




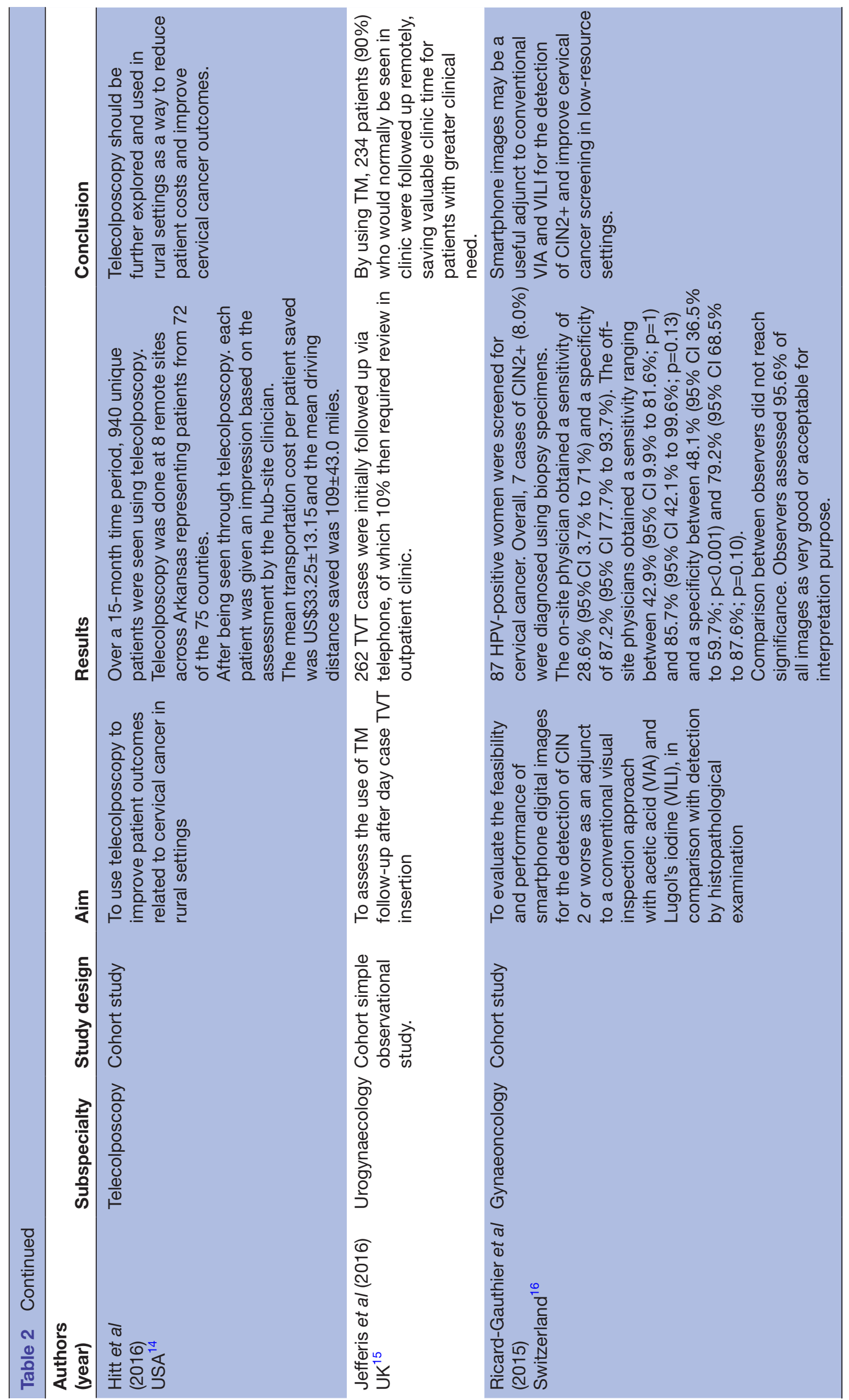




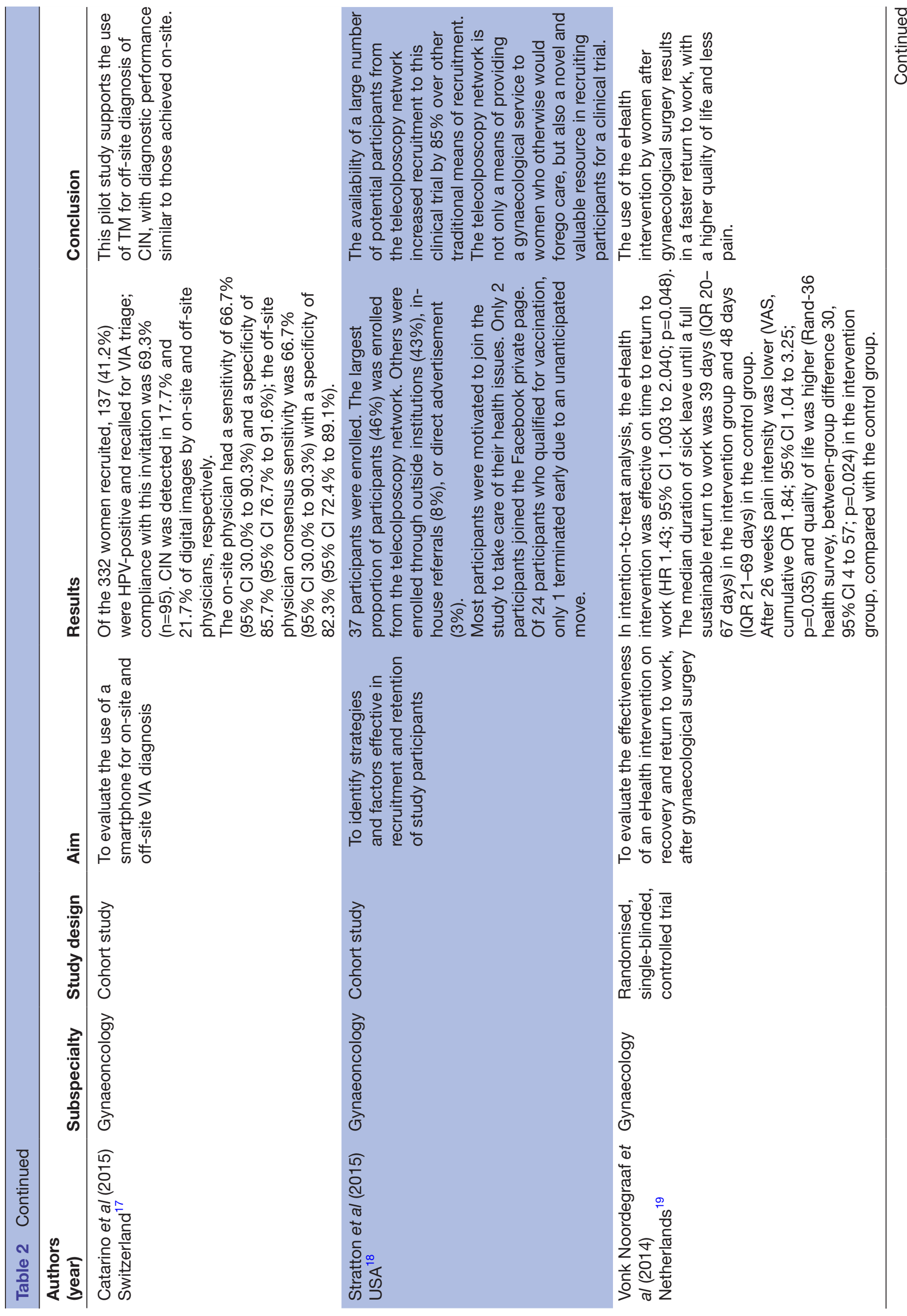


뜌

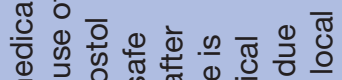

ह 0 잉

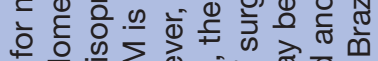

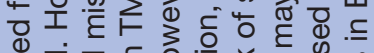

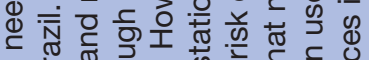

ब.

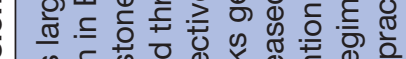

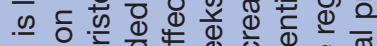

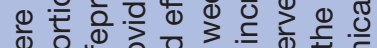

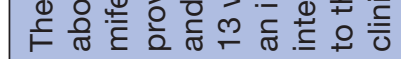

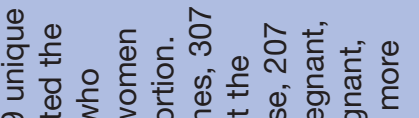

ᄋ

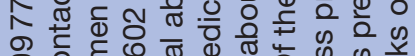

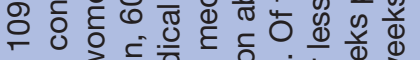

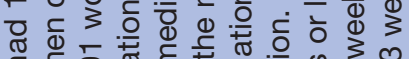

ฮ ब

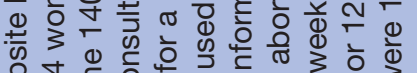

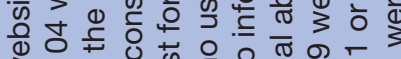

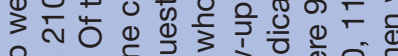

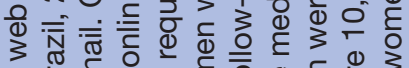

ठ

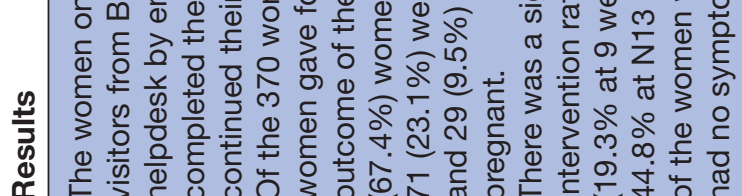

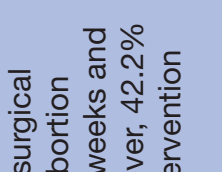

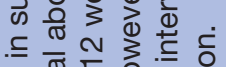

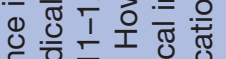

के है

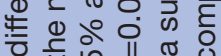

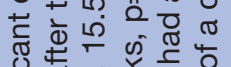

苋范

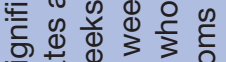

के एँ

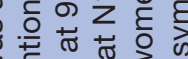

告

뜬 긍 농 $\frac{0}{0}$

당 0

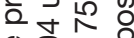

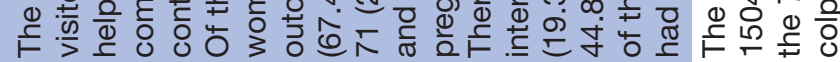

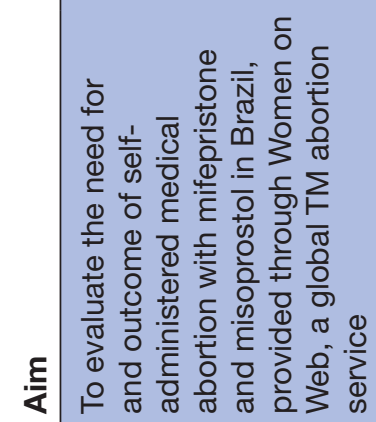

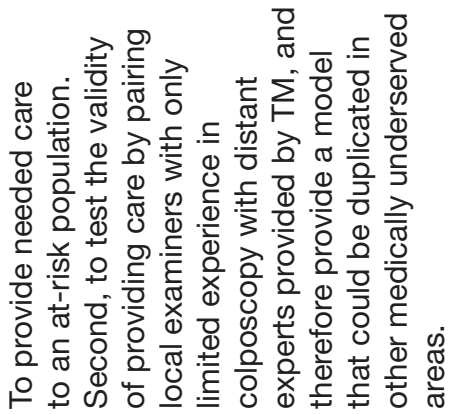

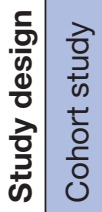

훌

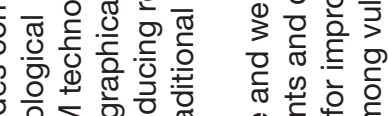

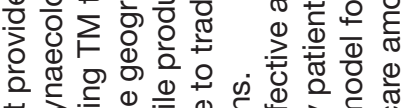

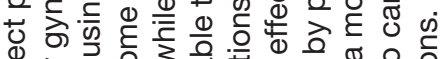

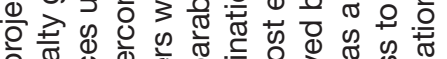

क

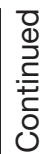

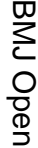

$\stackrel{\vec{F}}{\stackrel{9}{+}}$

음

는

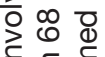

인

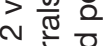

Ф)

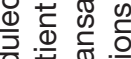

妾

o $\subseteq$

क

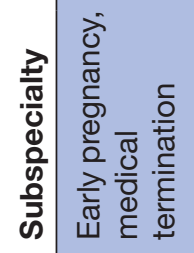

章 


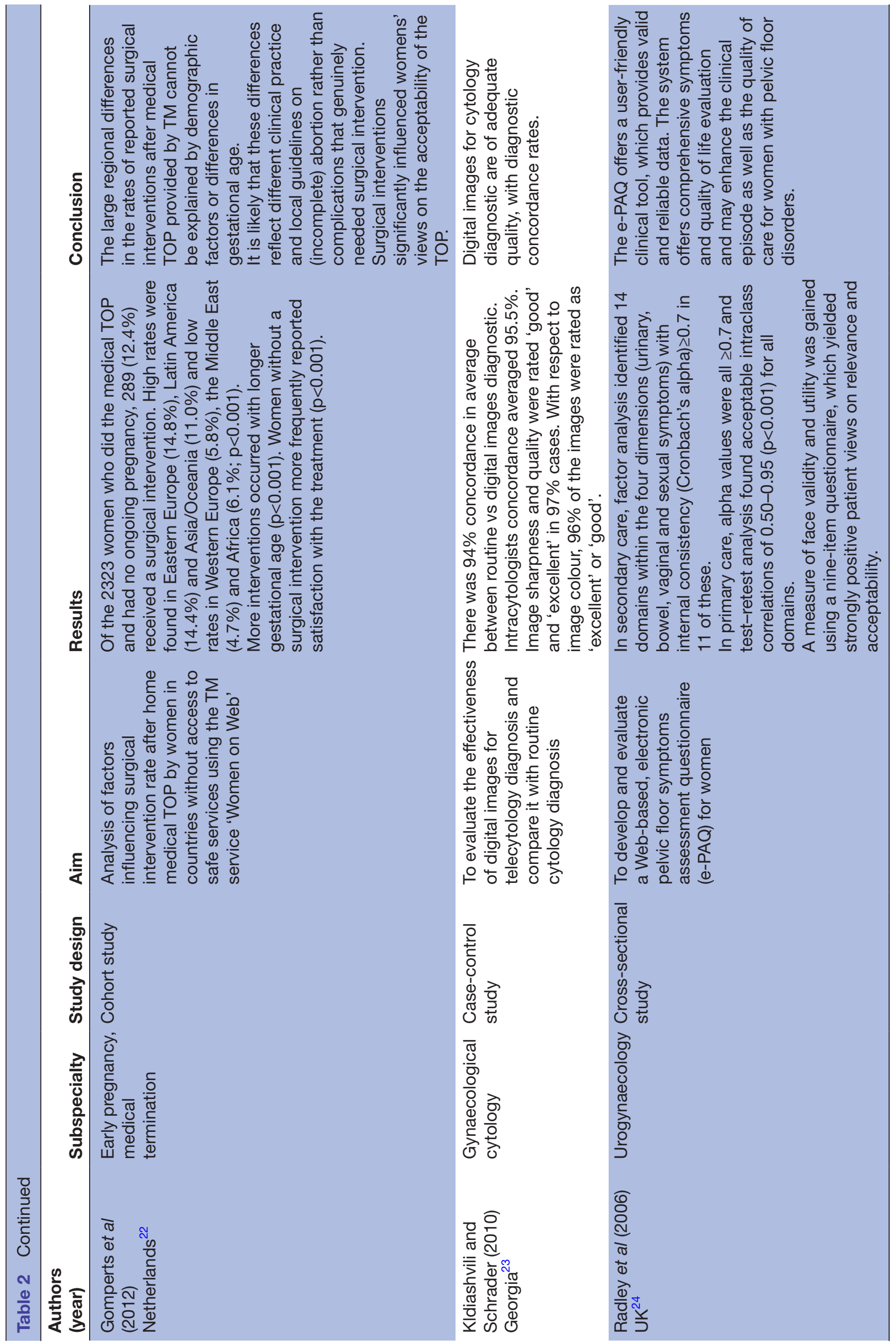




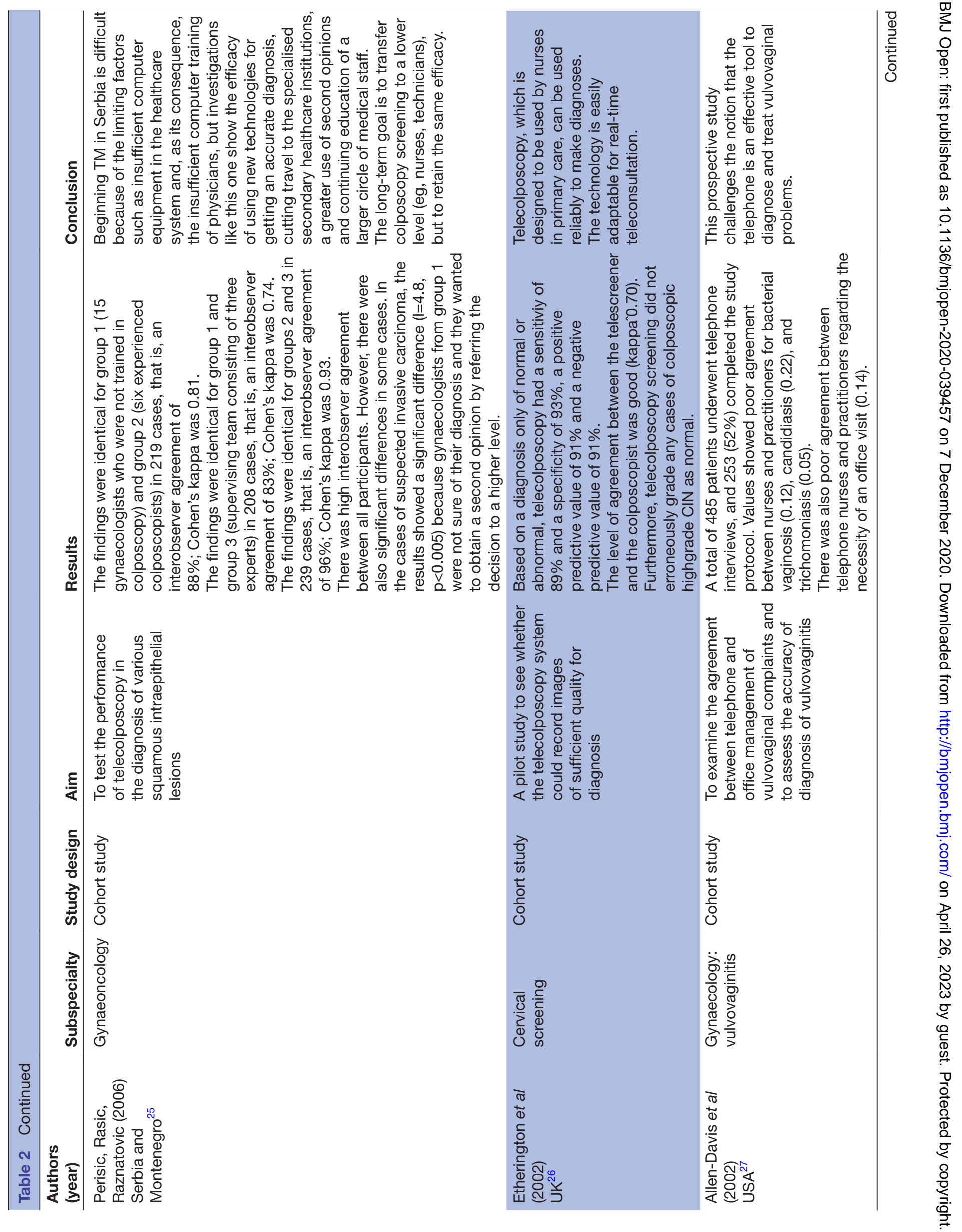




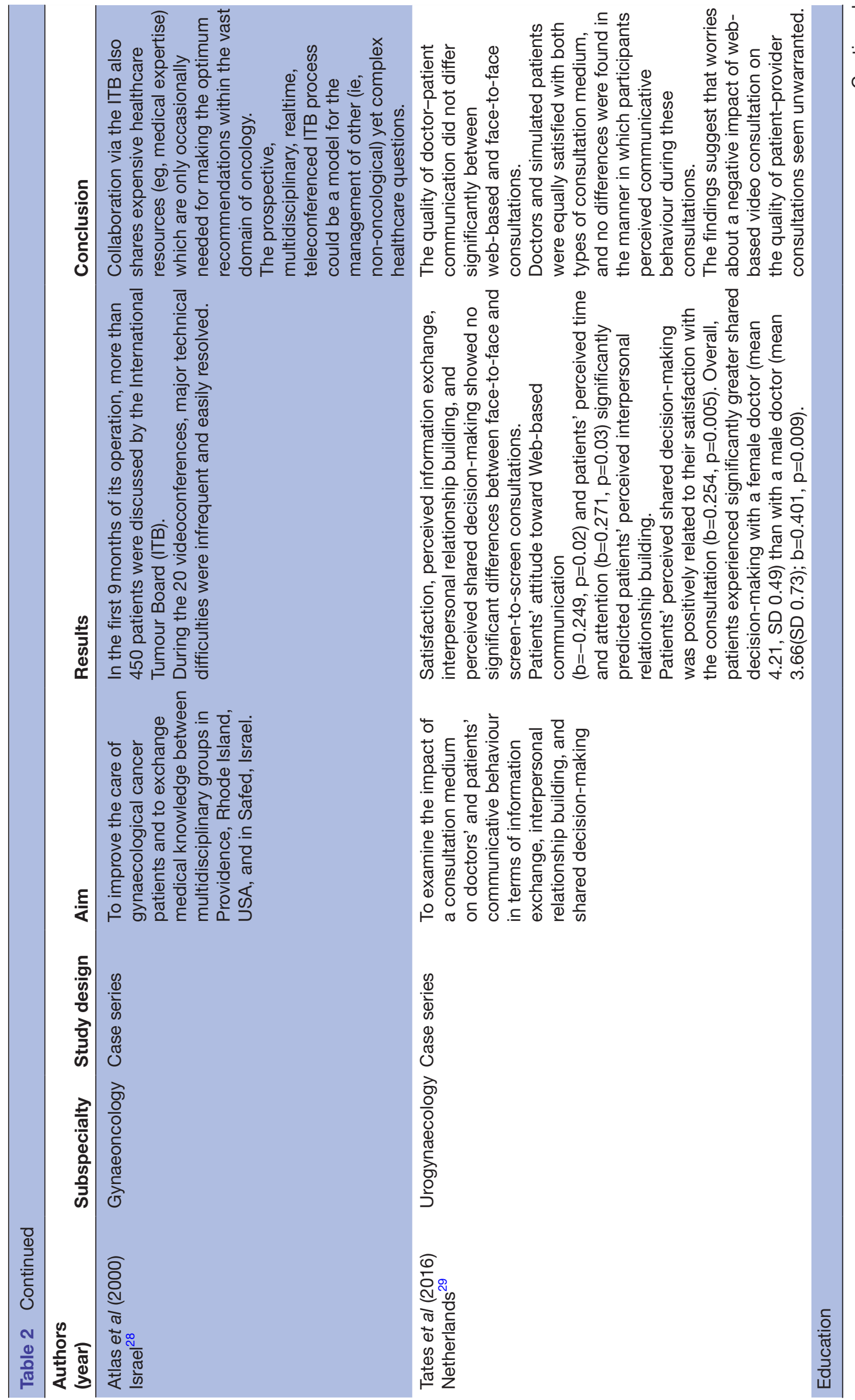




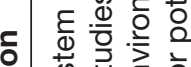

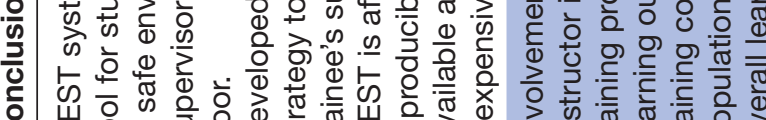

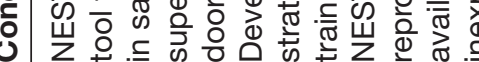

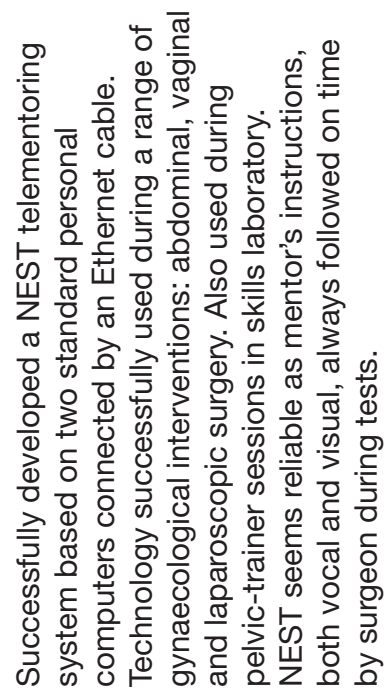

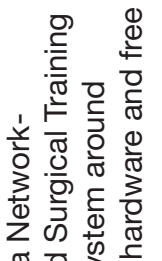

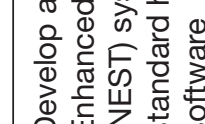

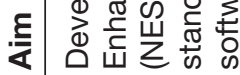

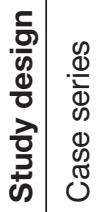

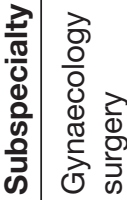

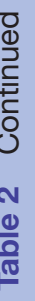

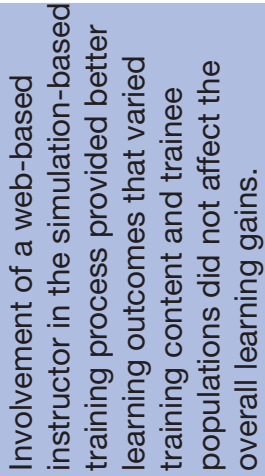

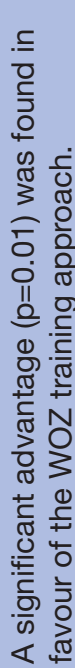

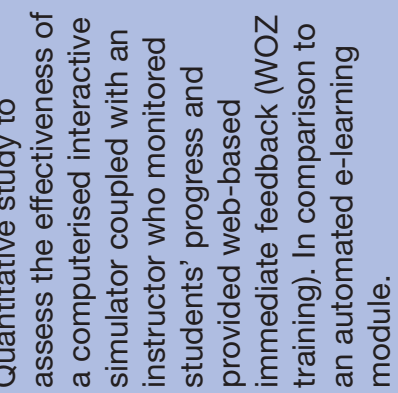

วิ

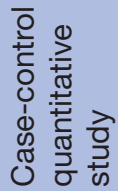

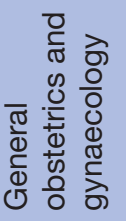

$\bar{\top}$

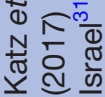

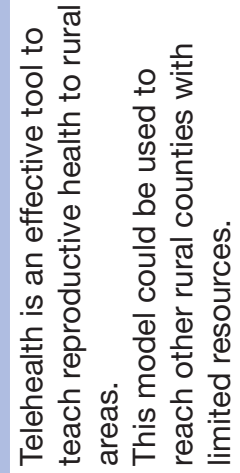

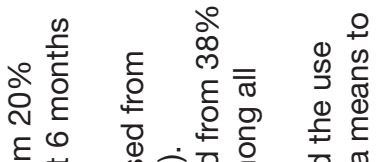

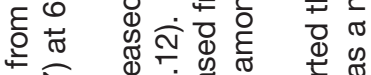
药 ه్

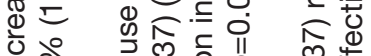
ㅇํㄷㅇㅝ ब.

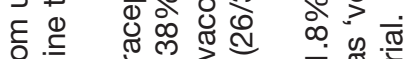

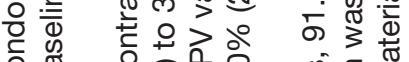

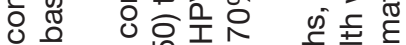

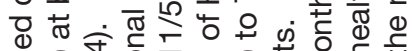

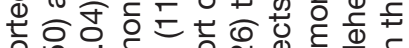

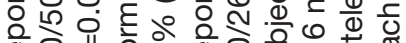

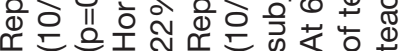

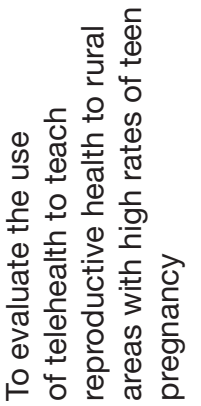

1) 政

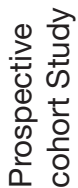

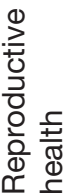

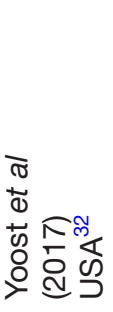




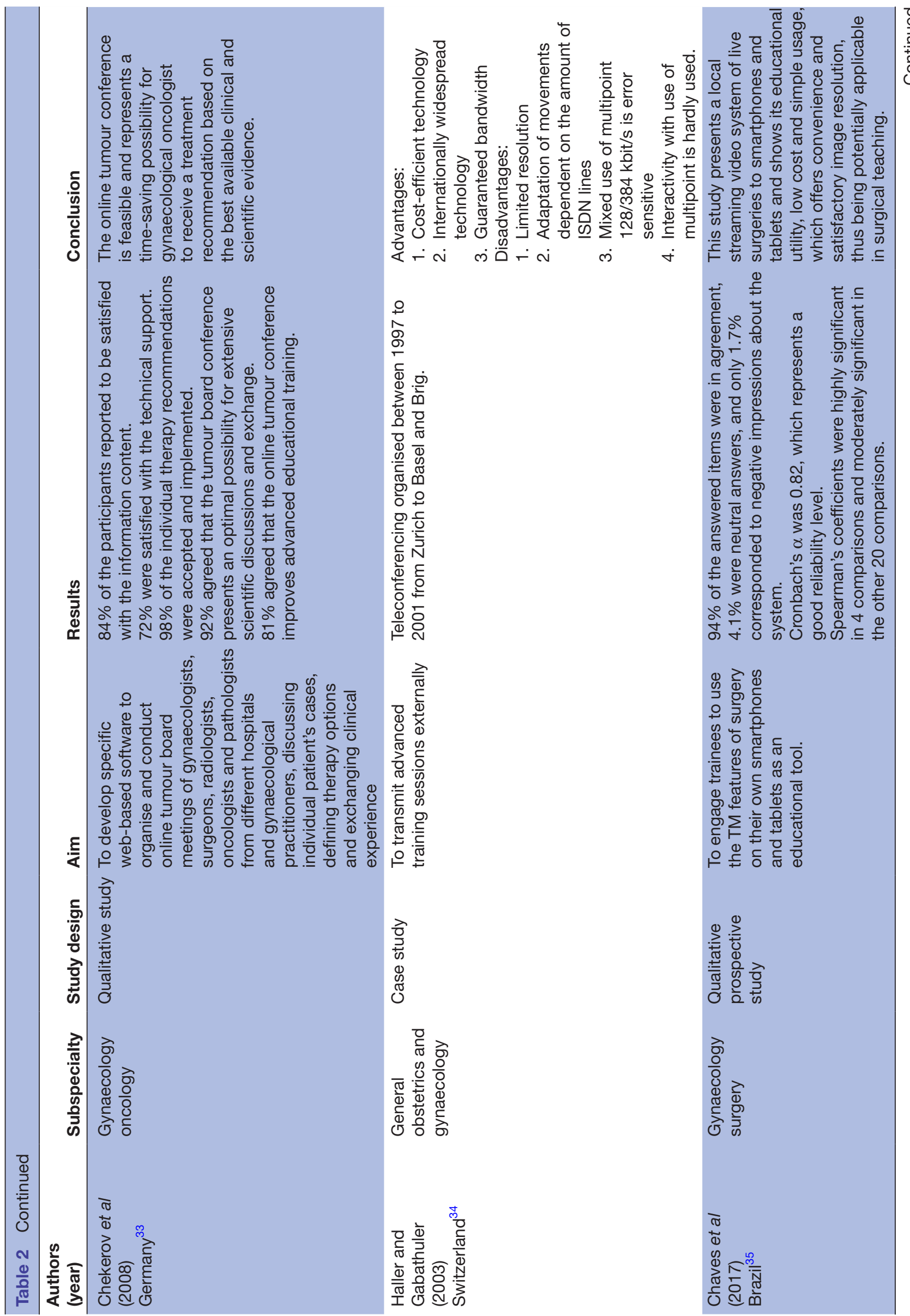

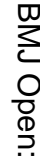

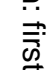

흠 .

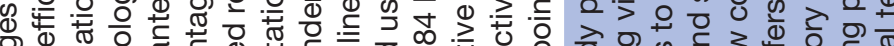

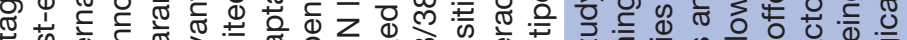

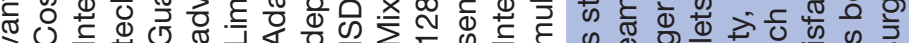

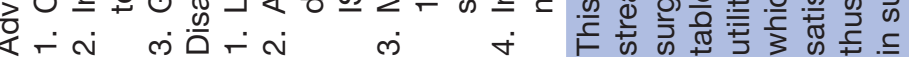

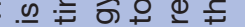


$\frac{2}{9}$
$\frac{0}{0}$
0
0
0

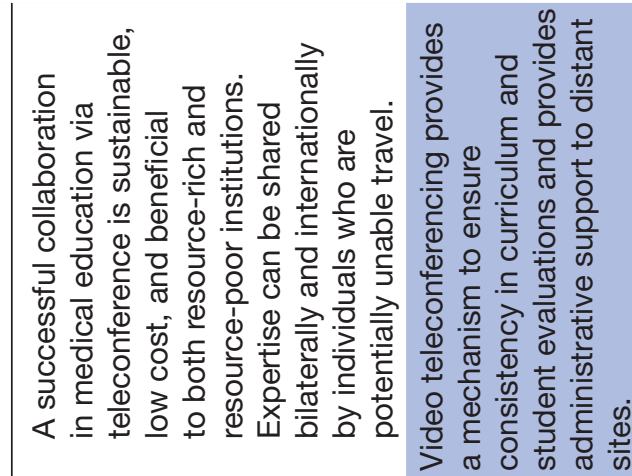

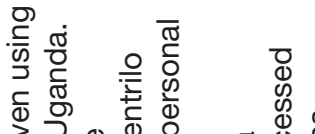

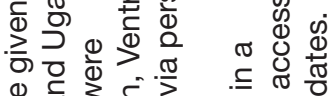

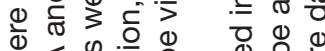

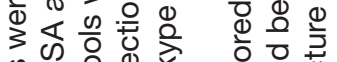

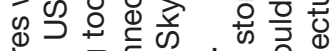

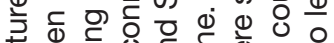

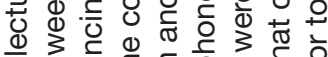

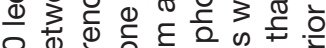

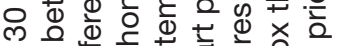

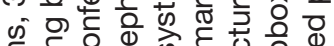
F 엉 के के क्ष

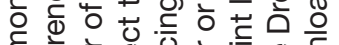

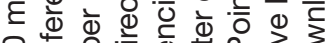
๑ 声

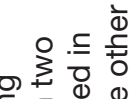

.

运

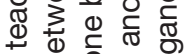
๘ 0 ¿

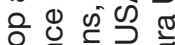
ब.

छ

安

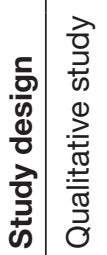

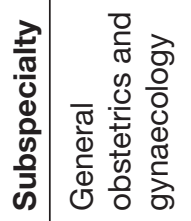

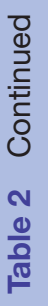

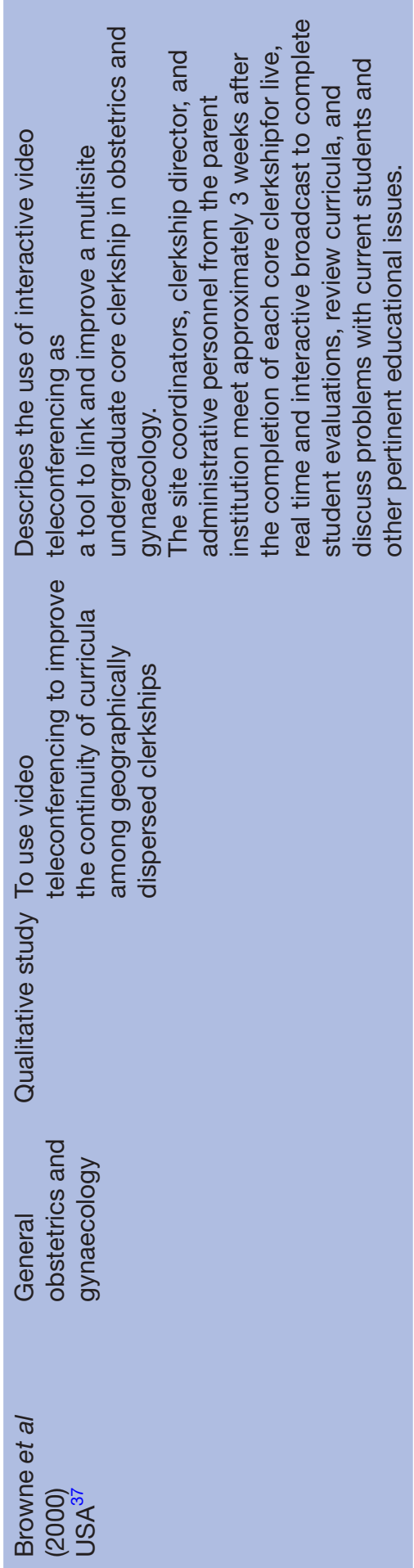

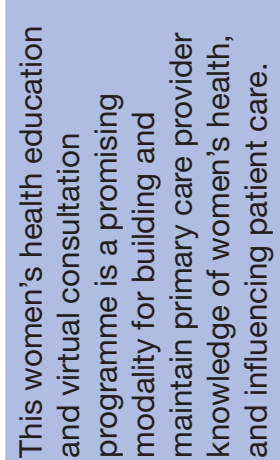

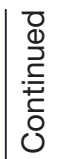

눙

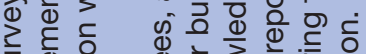

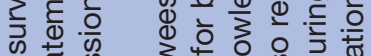

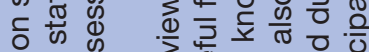

W

舟

फ 责恼

잉

ก․

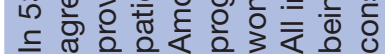

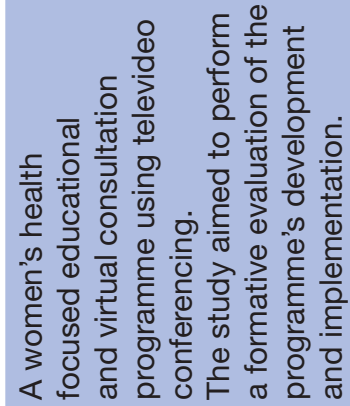

$\varangle$ 응

가

突

$\stackrel{0}{\geq}$

荧

$\frac{0}{3}$

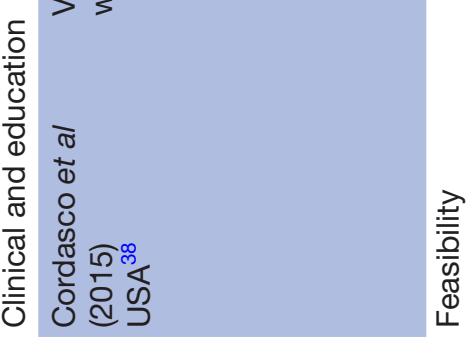




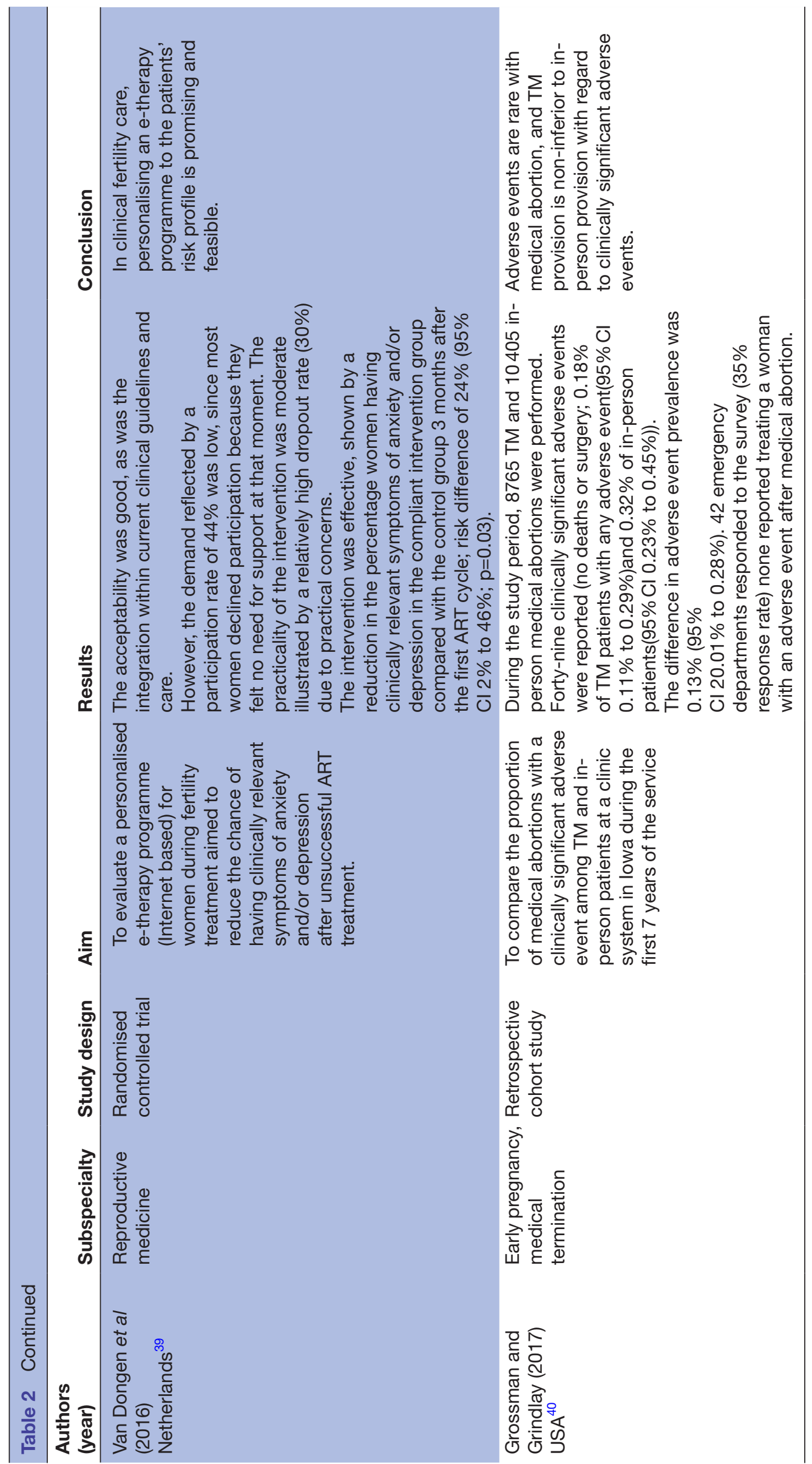




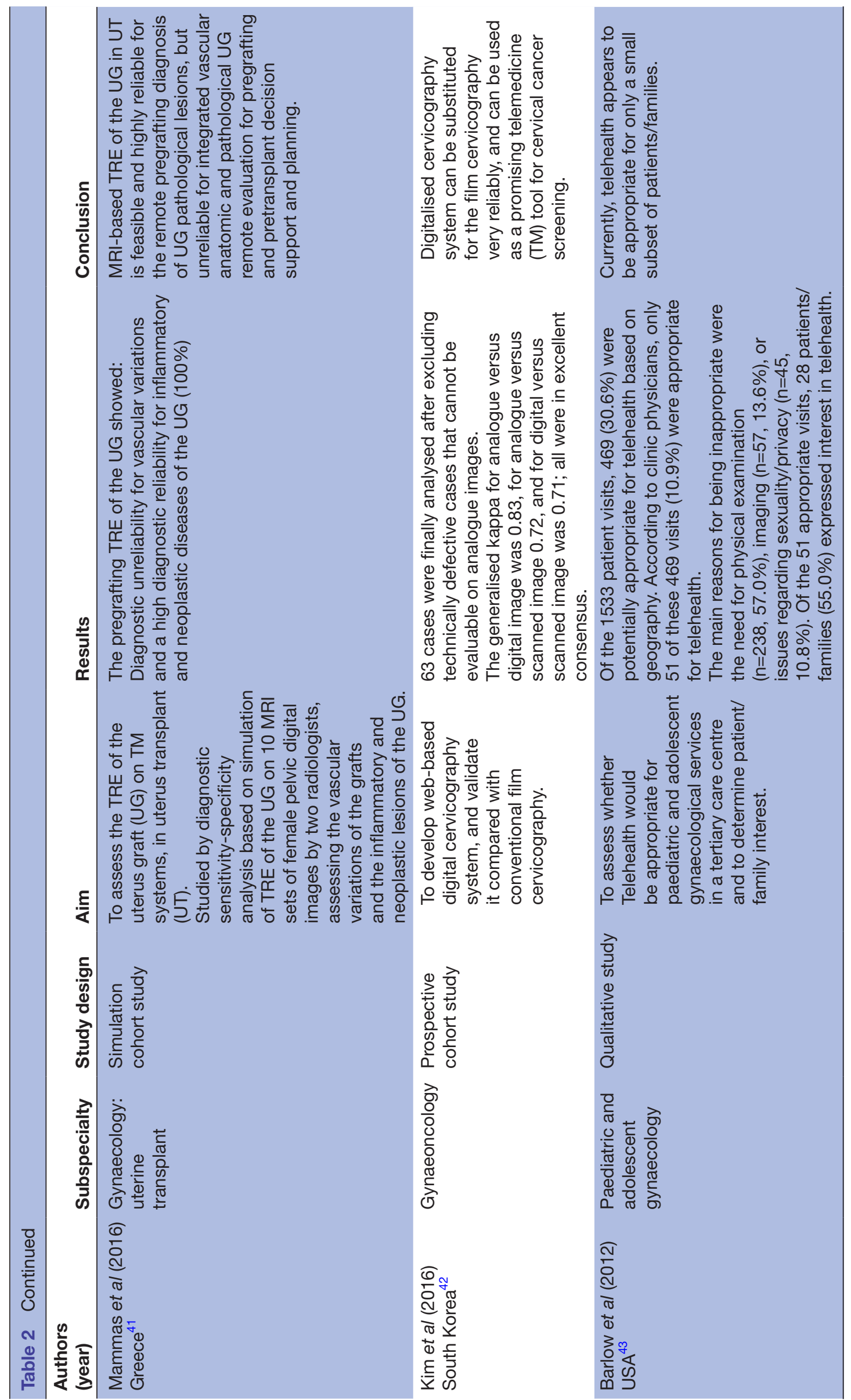




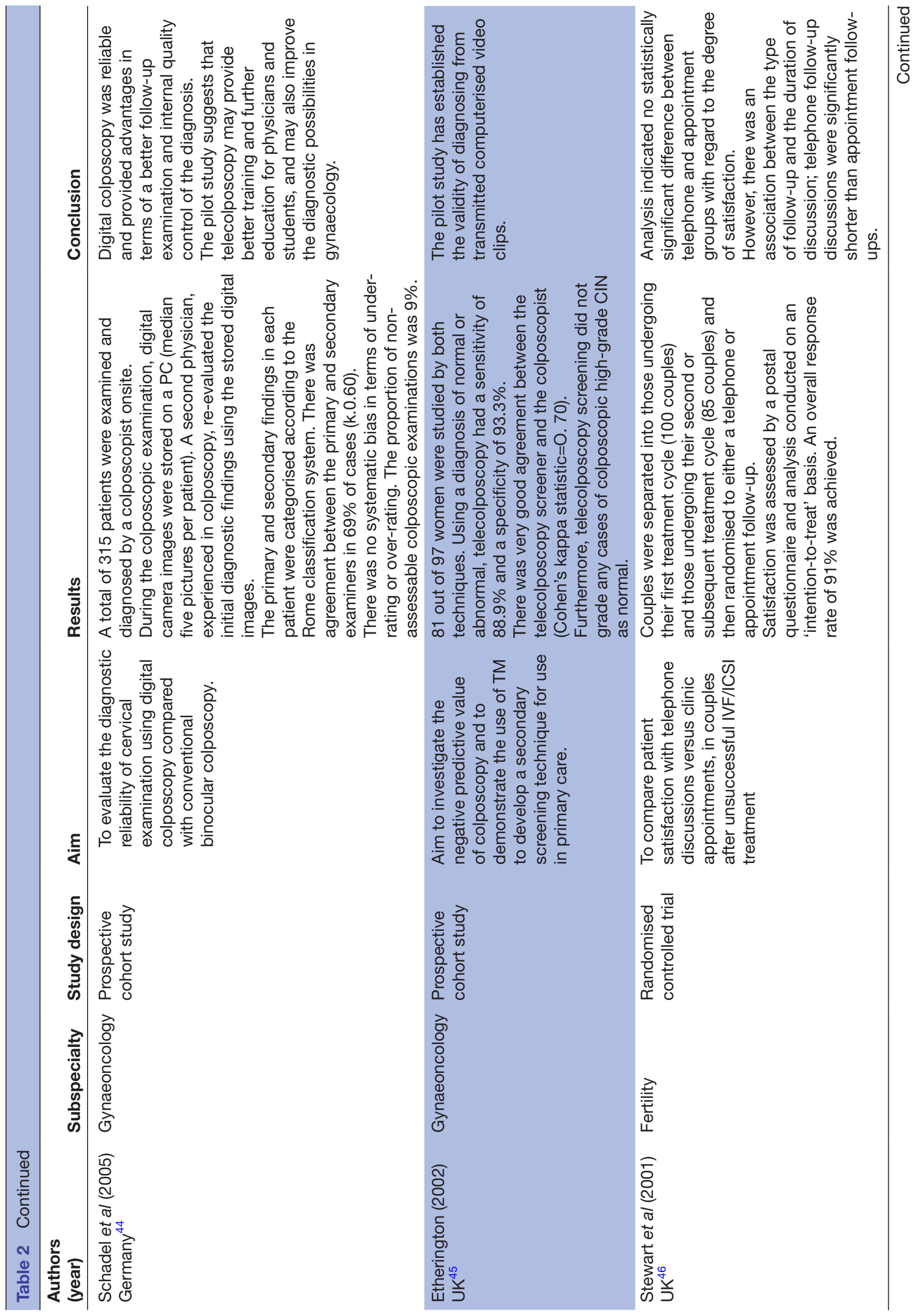



E \& क्ष.

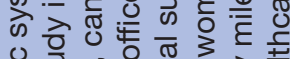

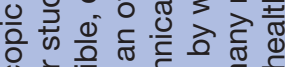

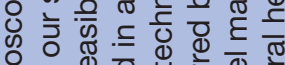
응.

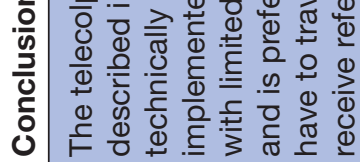

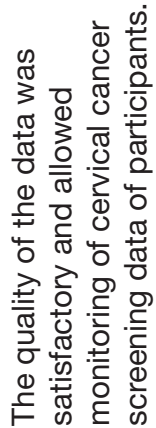

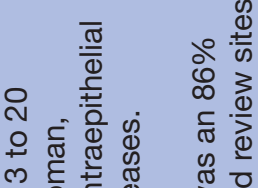

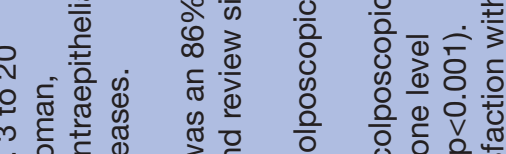

बं

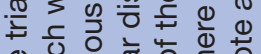

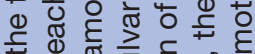

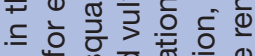

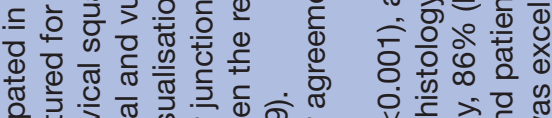

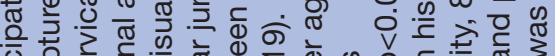

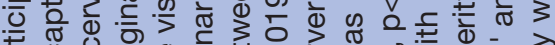

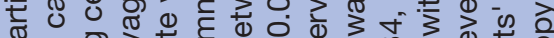

$\varrho$ 인.

Ф

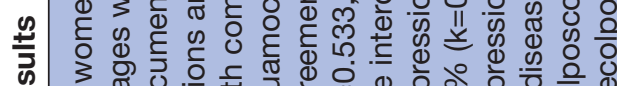

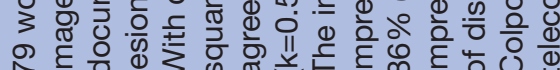

灵
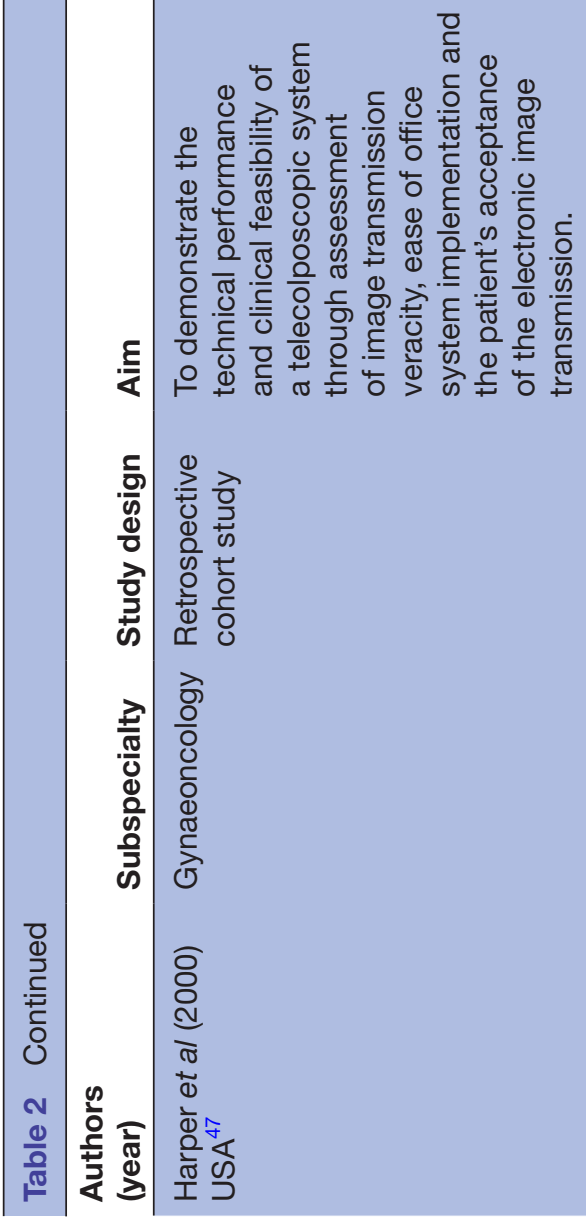

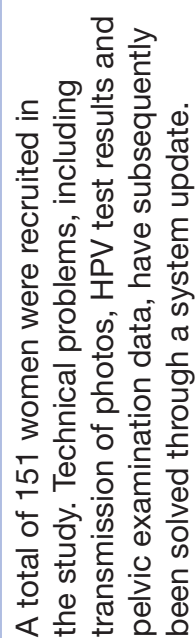

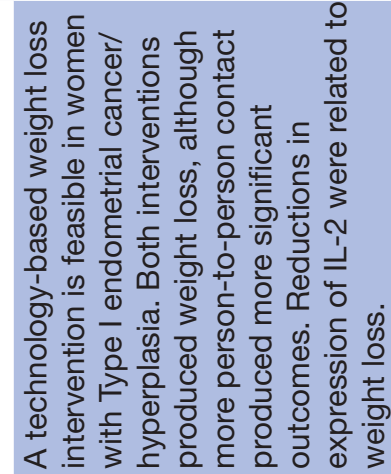

齐

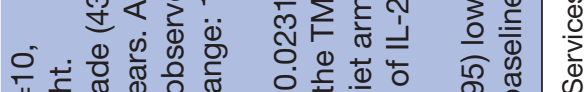

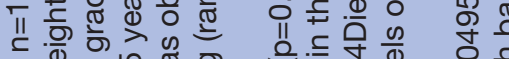

$\because 30$

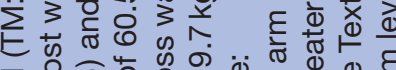

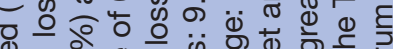

क人

ह

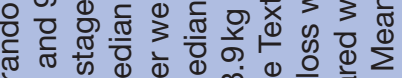

बे

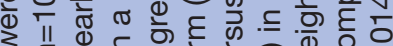

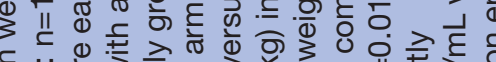

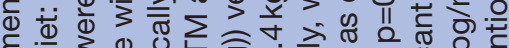

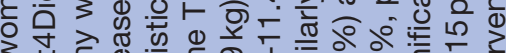

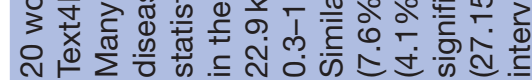
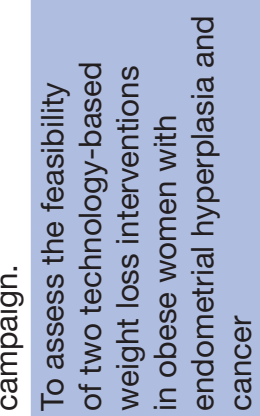

ก

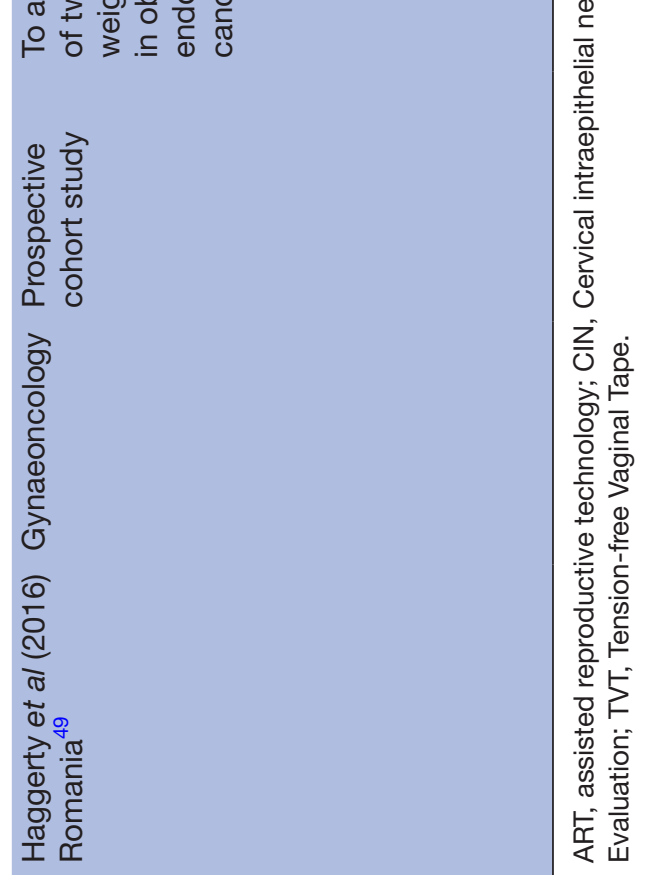




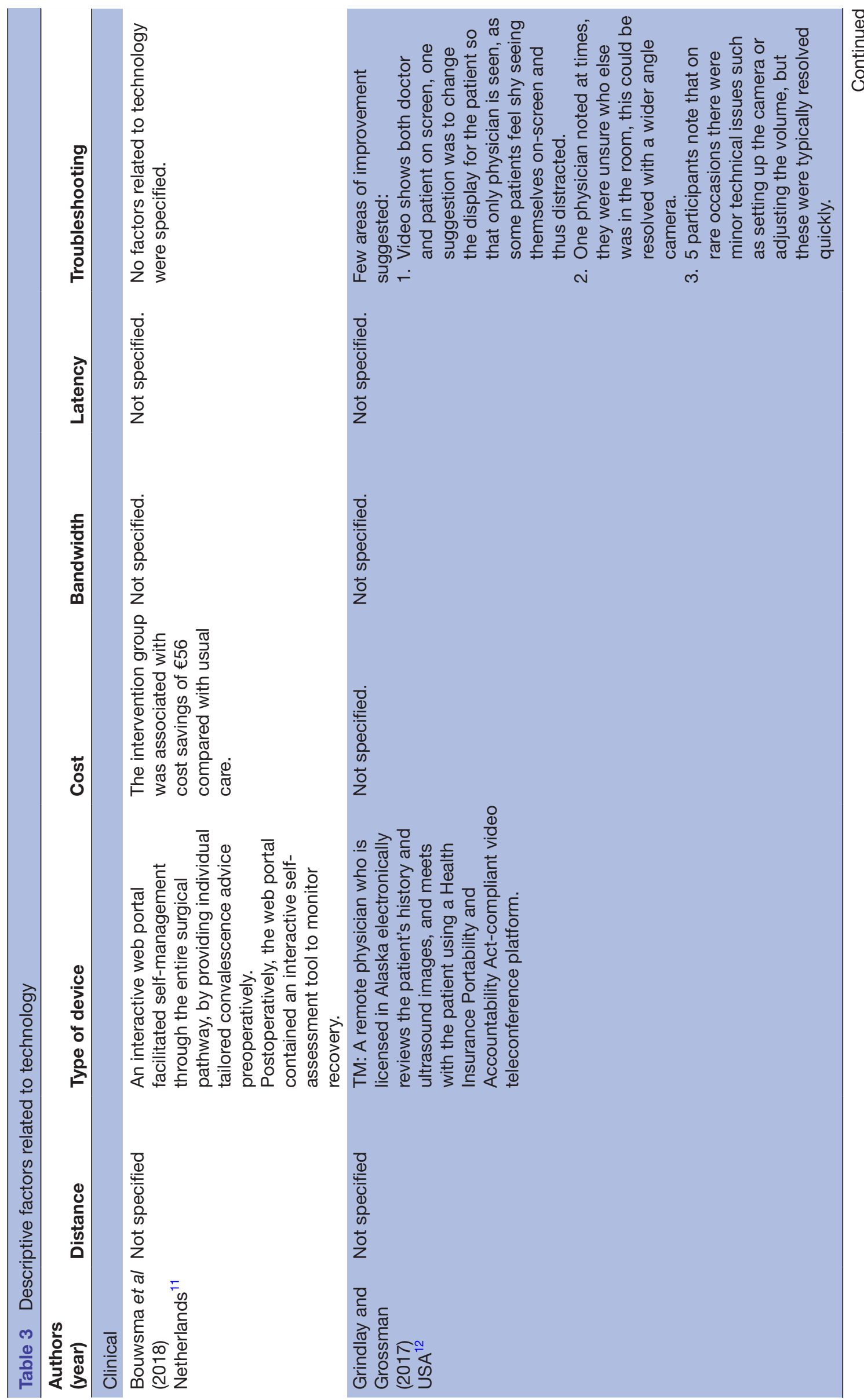




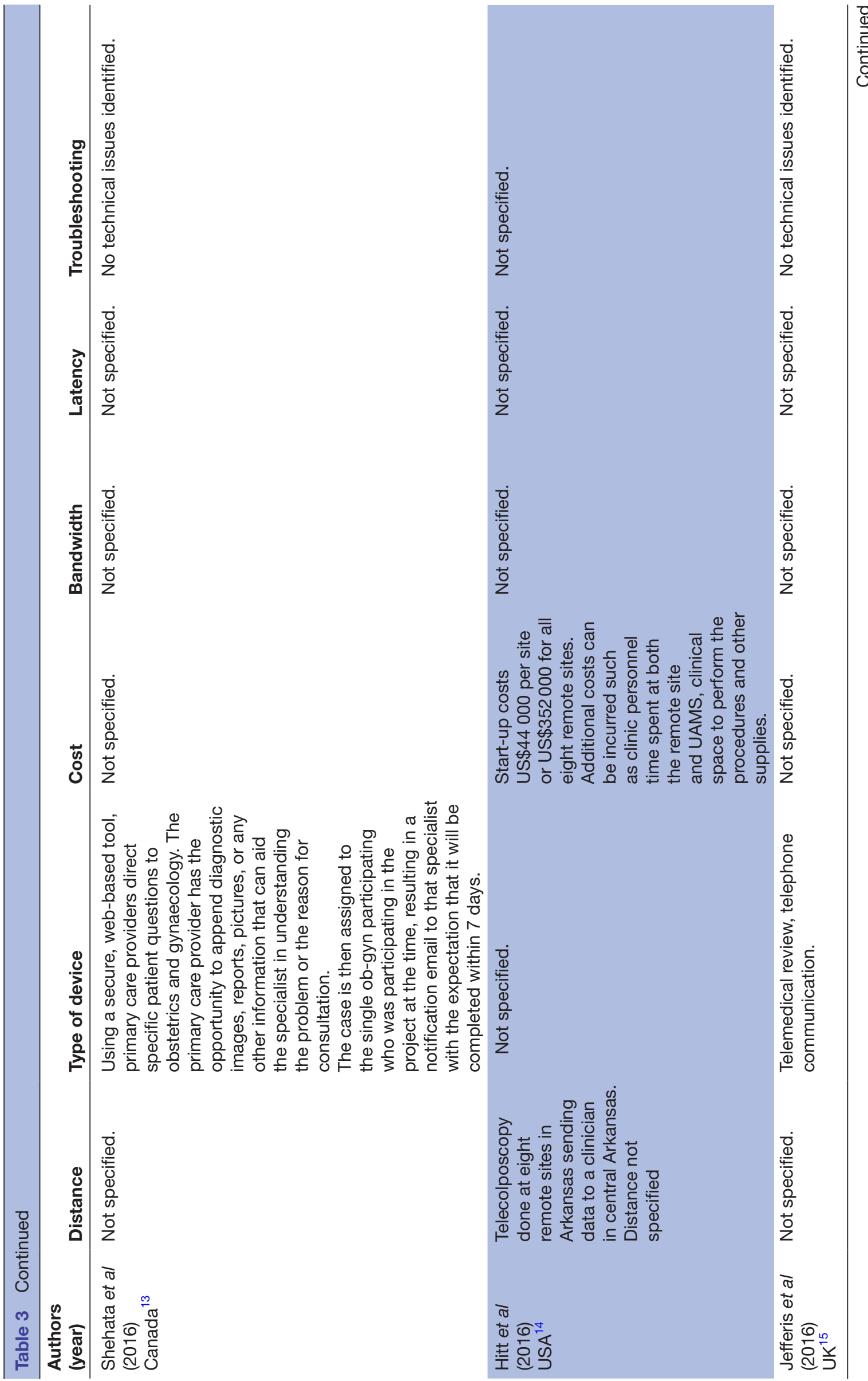




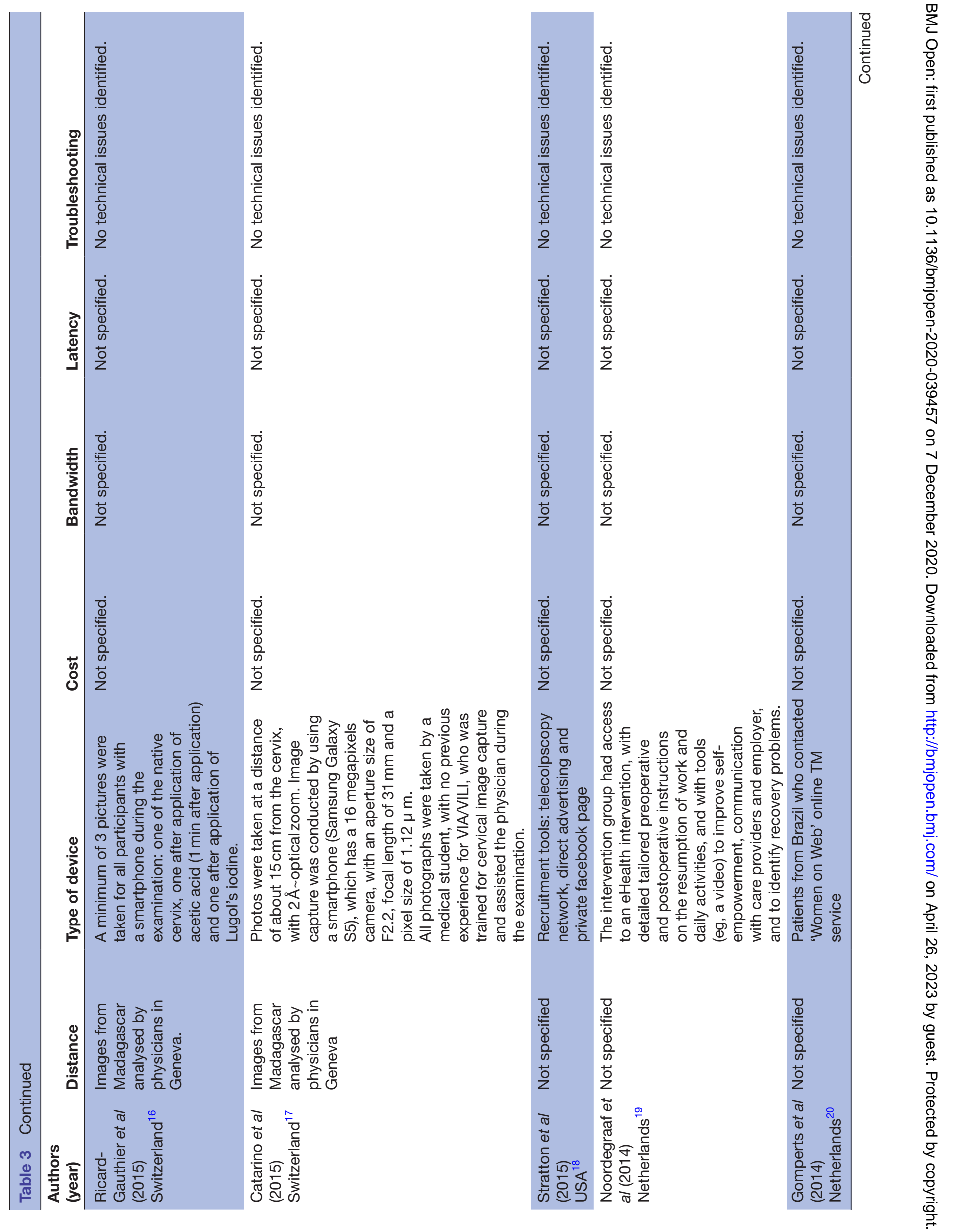




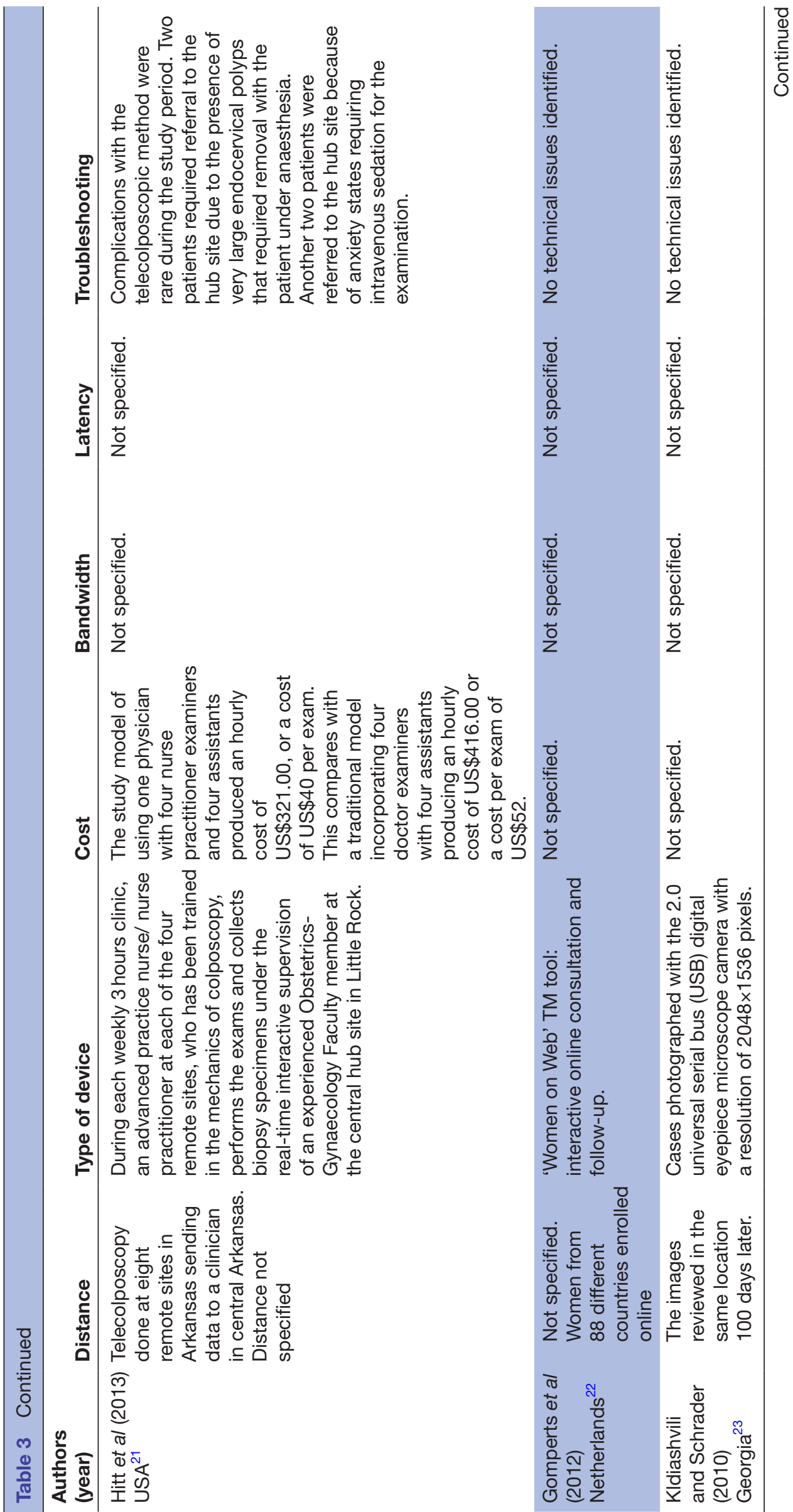




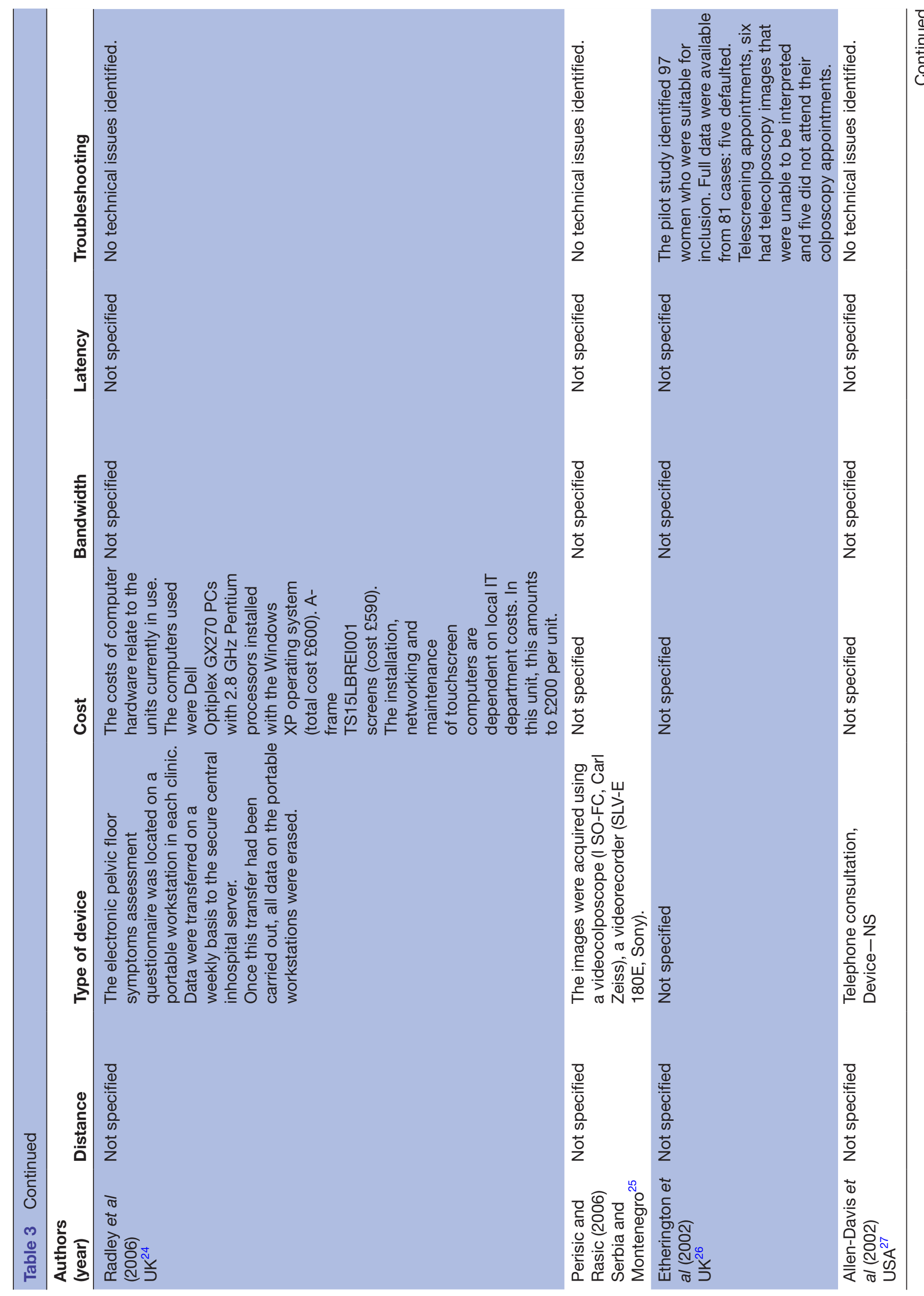




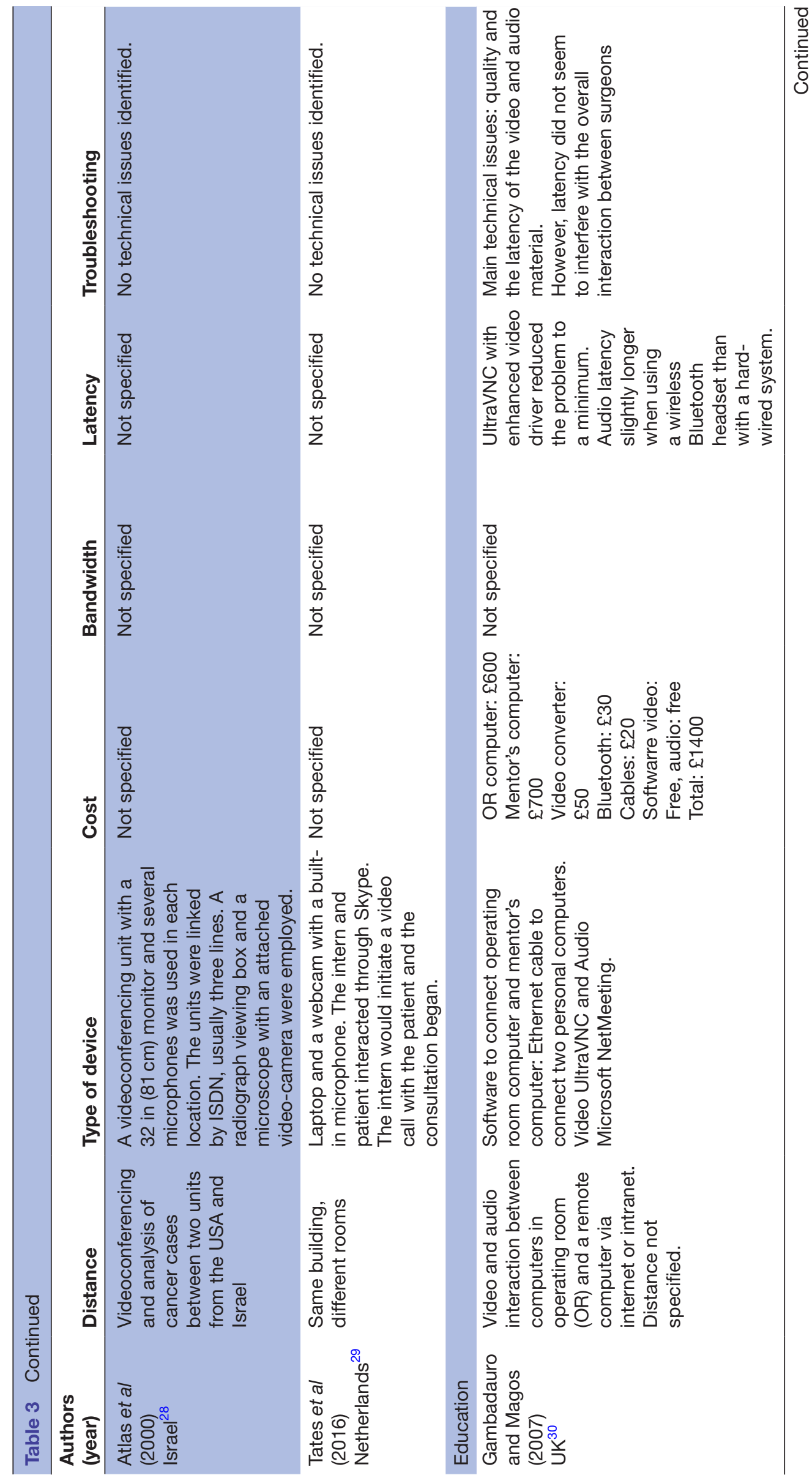




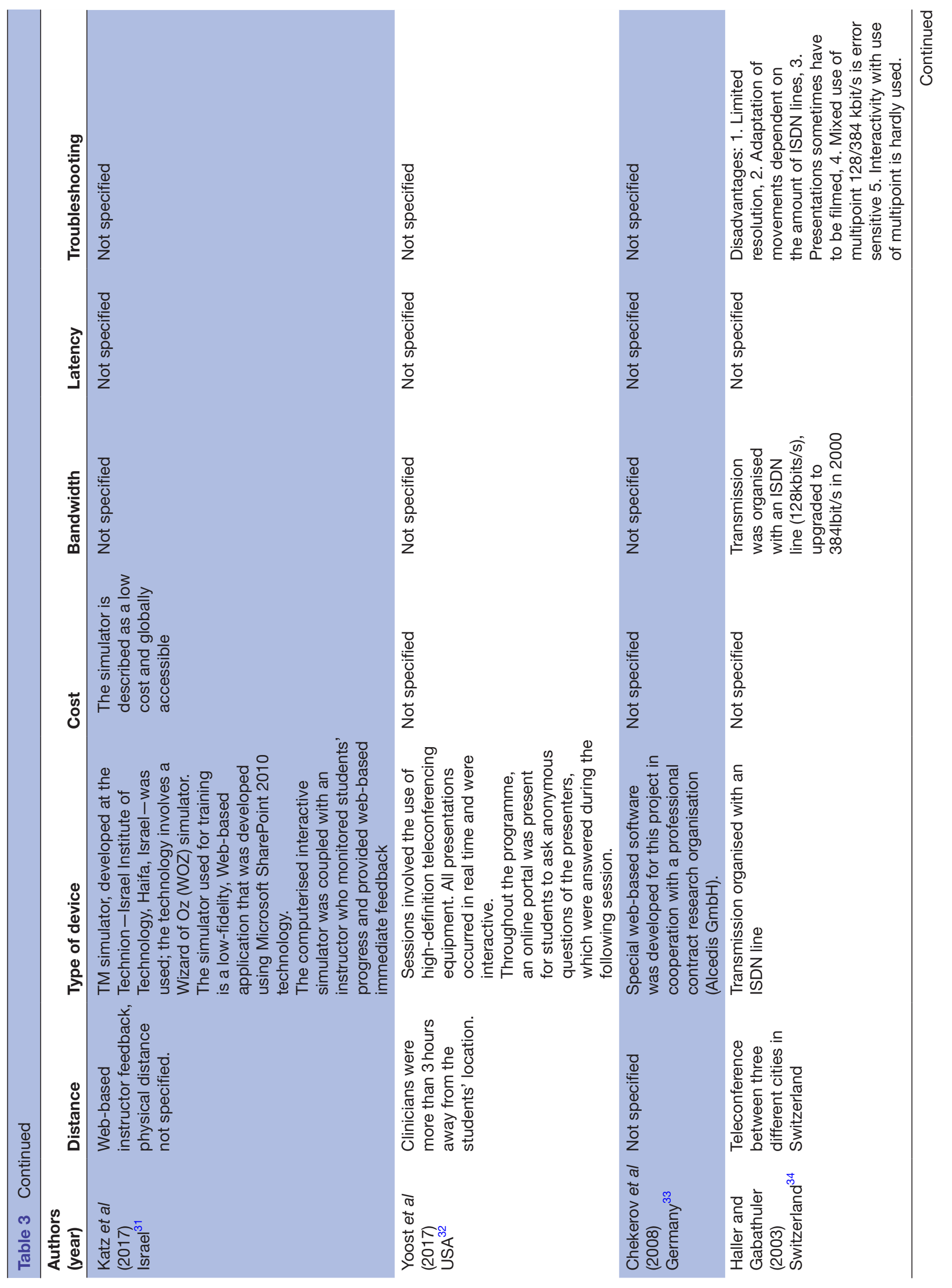



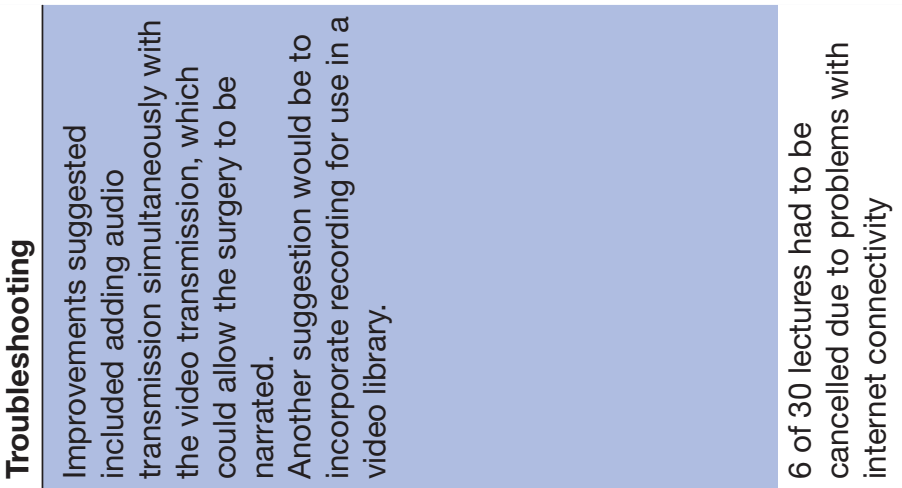

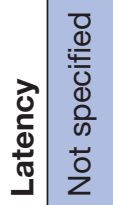

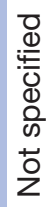

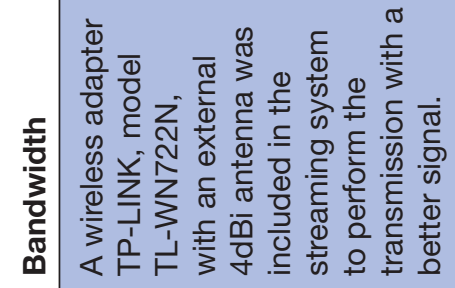

i

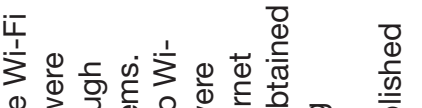

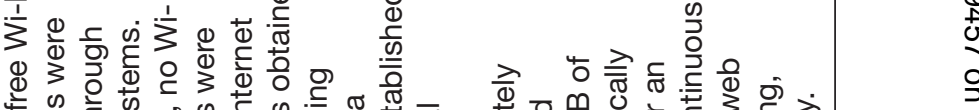

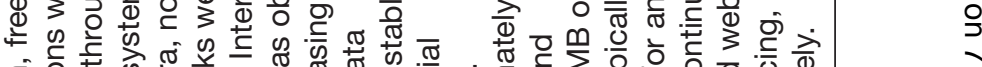

ᄃं.

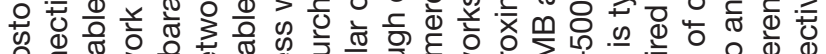

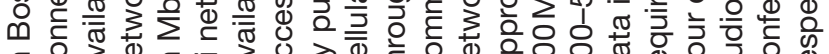

$\frac{5}{0} \frac{\grave{d}}{\partial}$

ॠิ

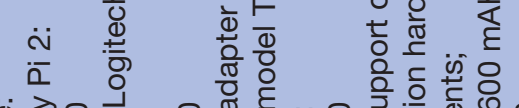

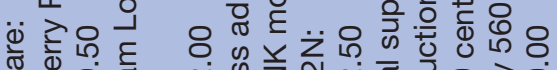

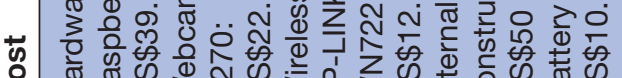

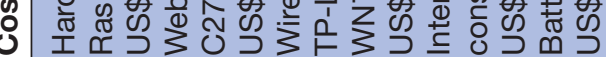

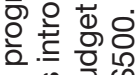

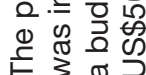
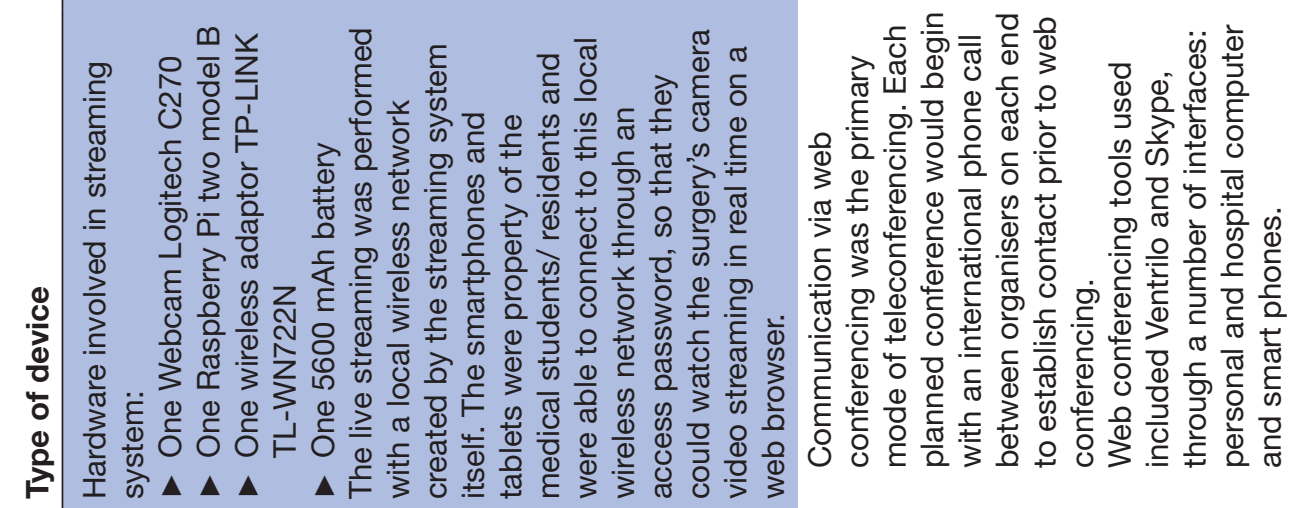

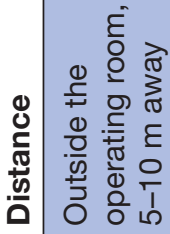
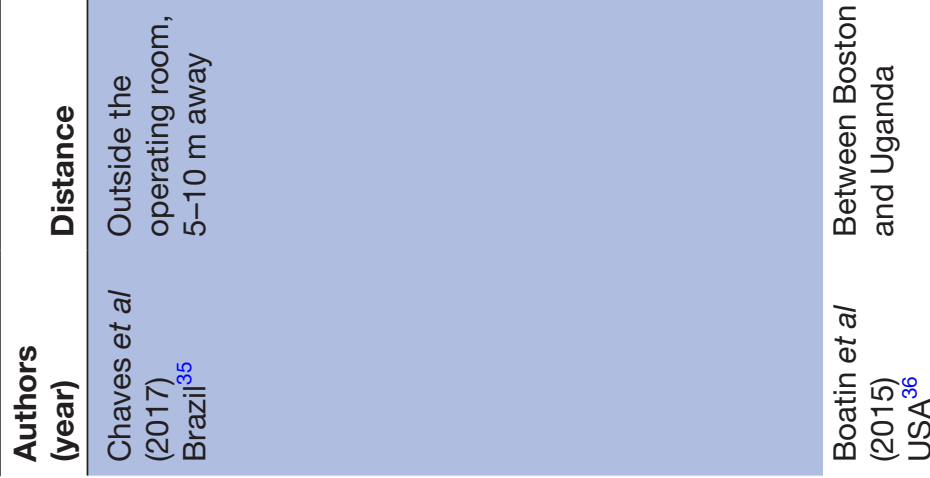


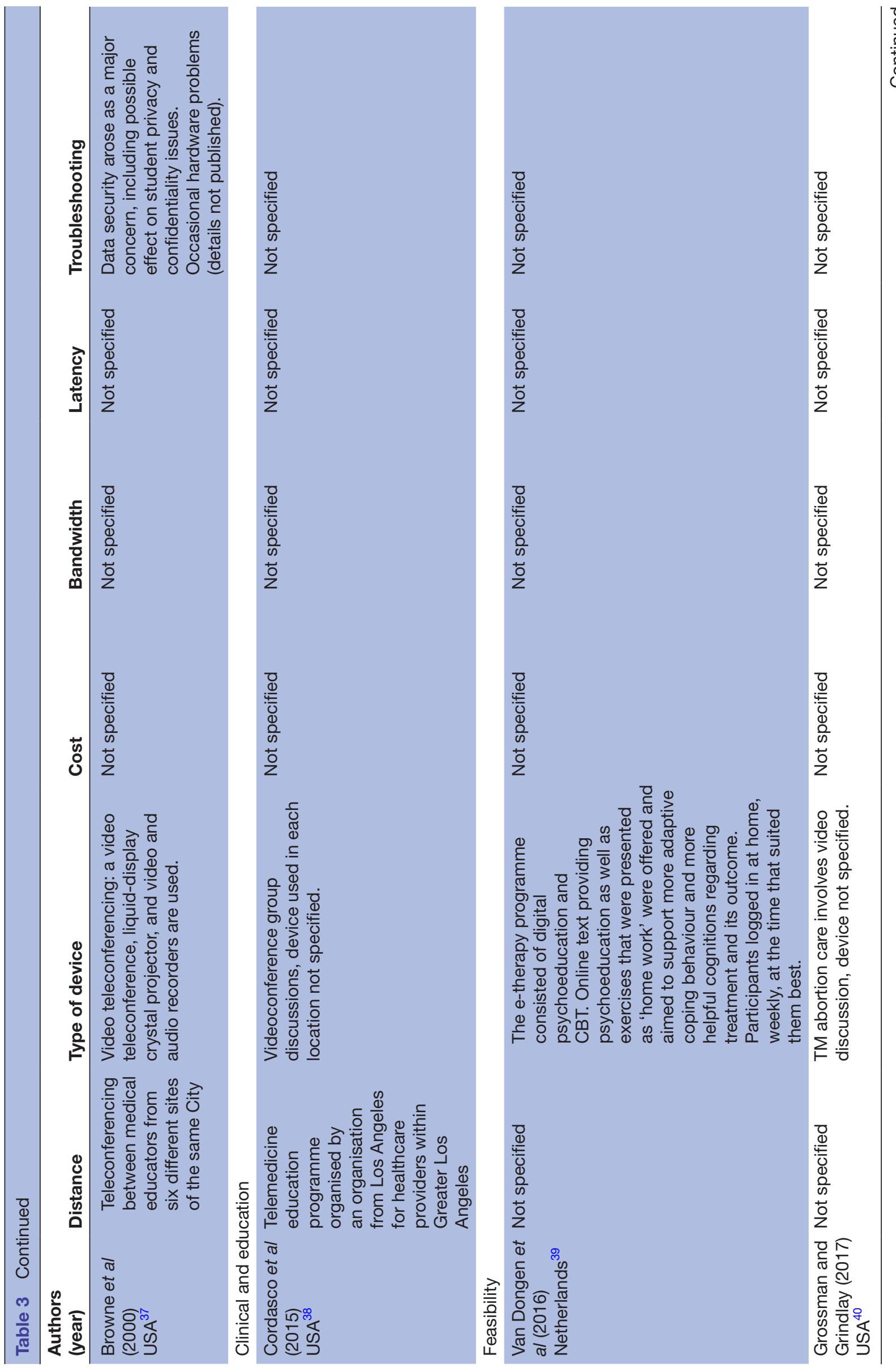




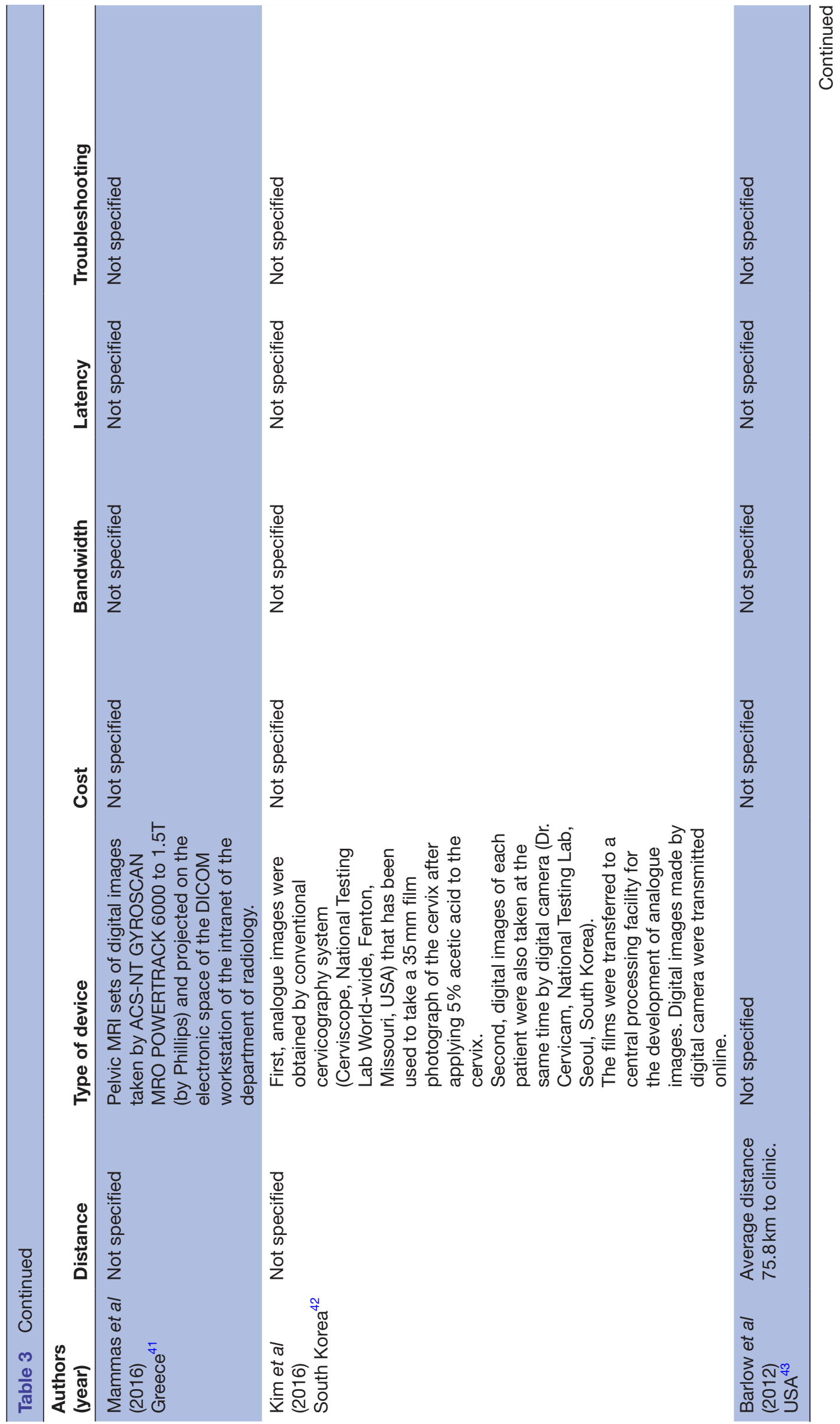




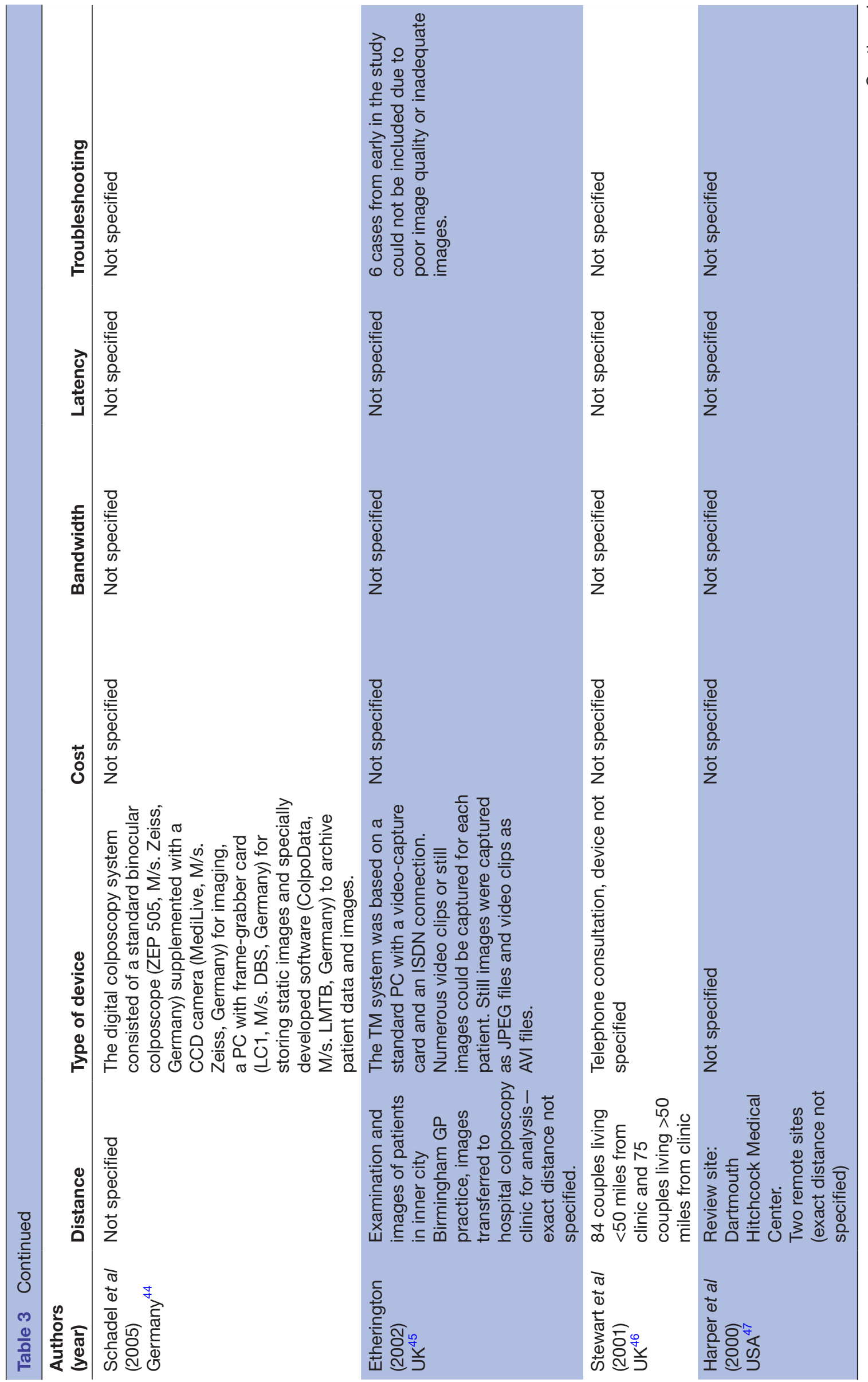




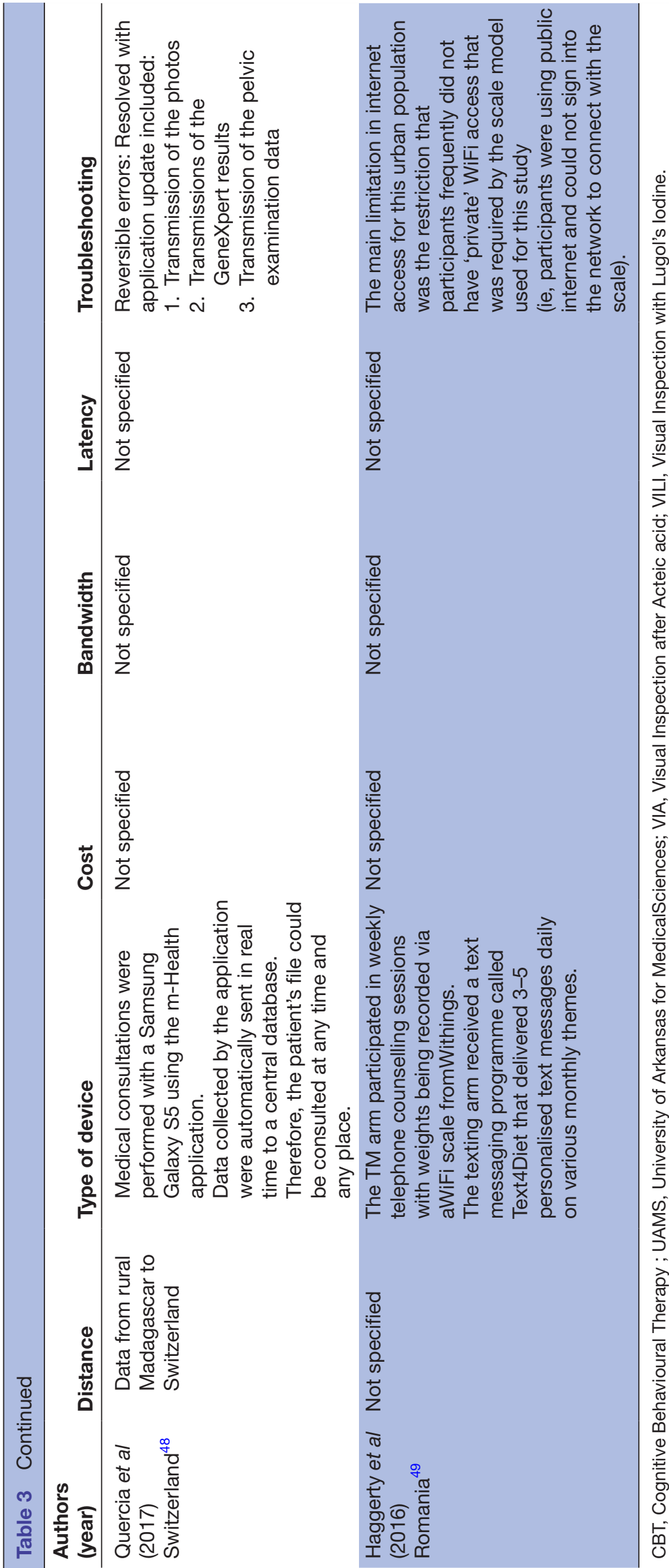




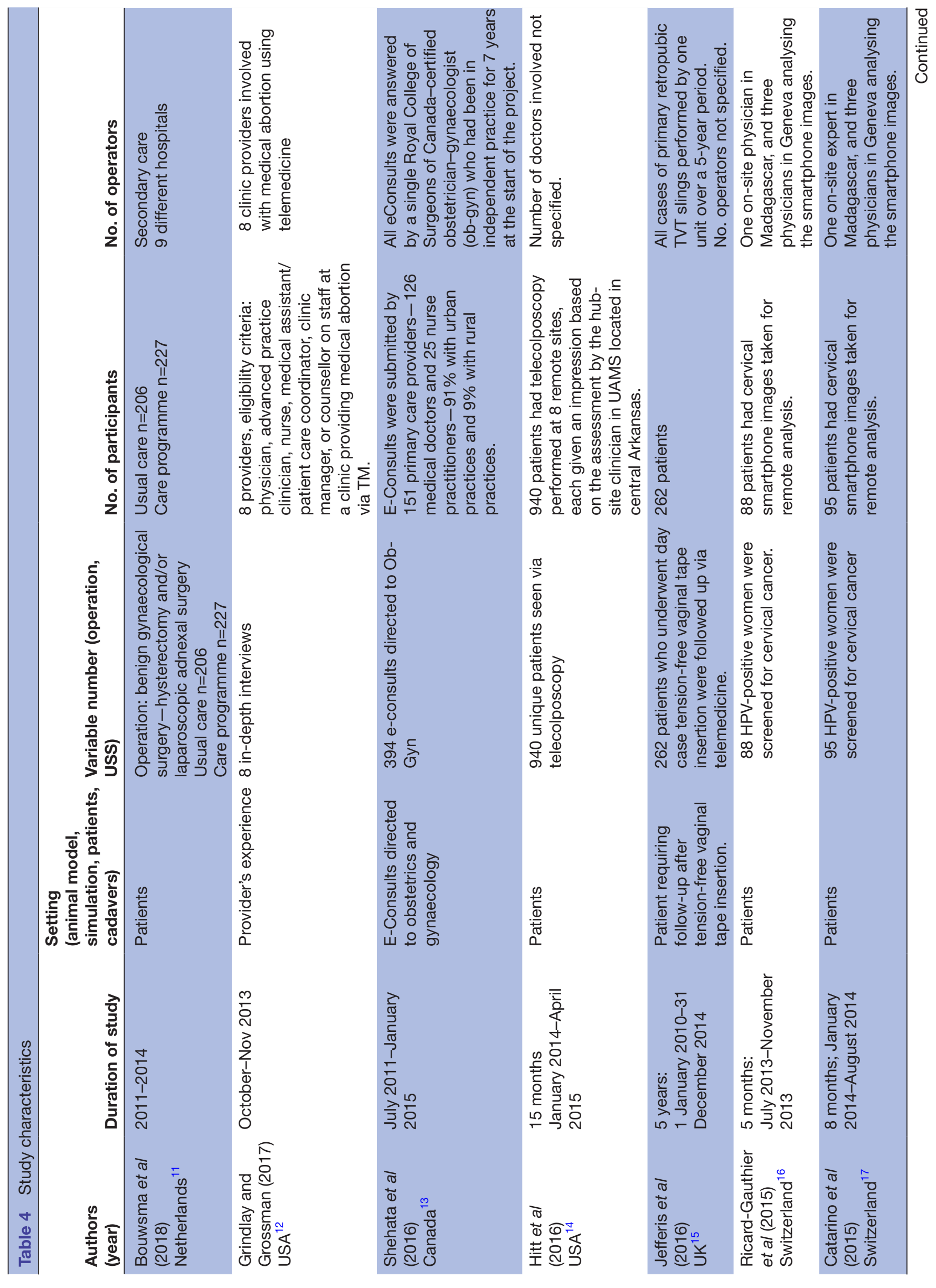



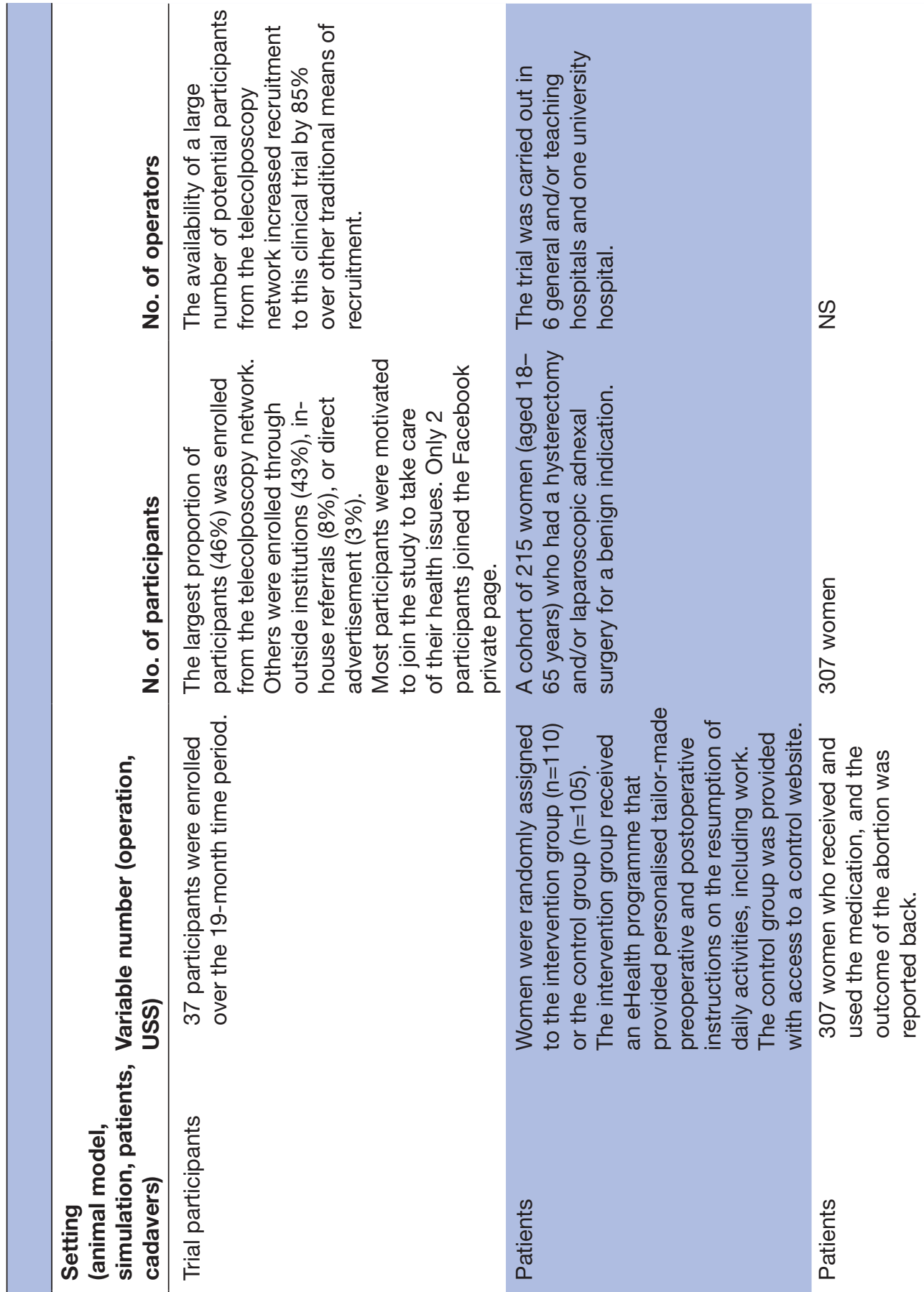

t.

$\frac{10}{20}$

ᄂ

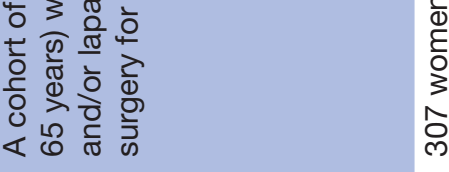

$\frac{\stackrel{\infty}{\frac{\infty}{0}}}{\frac{\infty}{\pi}}$

$\stackrel{\oplus}{ \pm}$
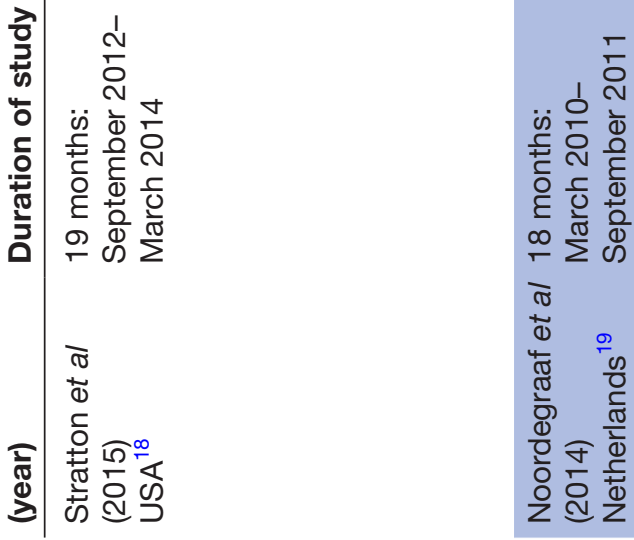

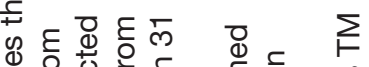

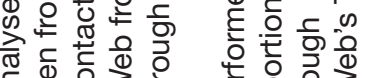

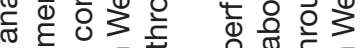

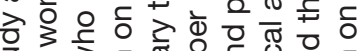
类

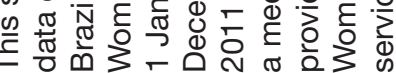



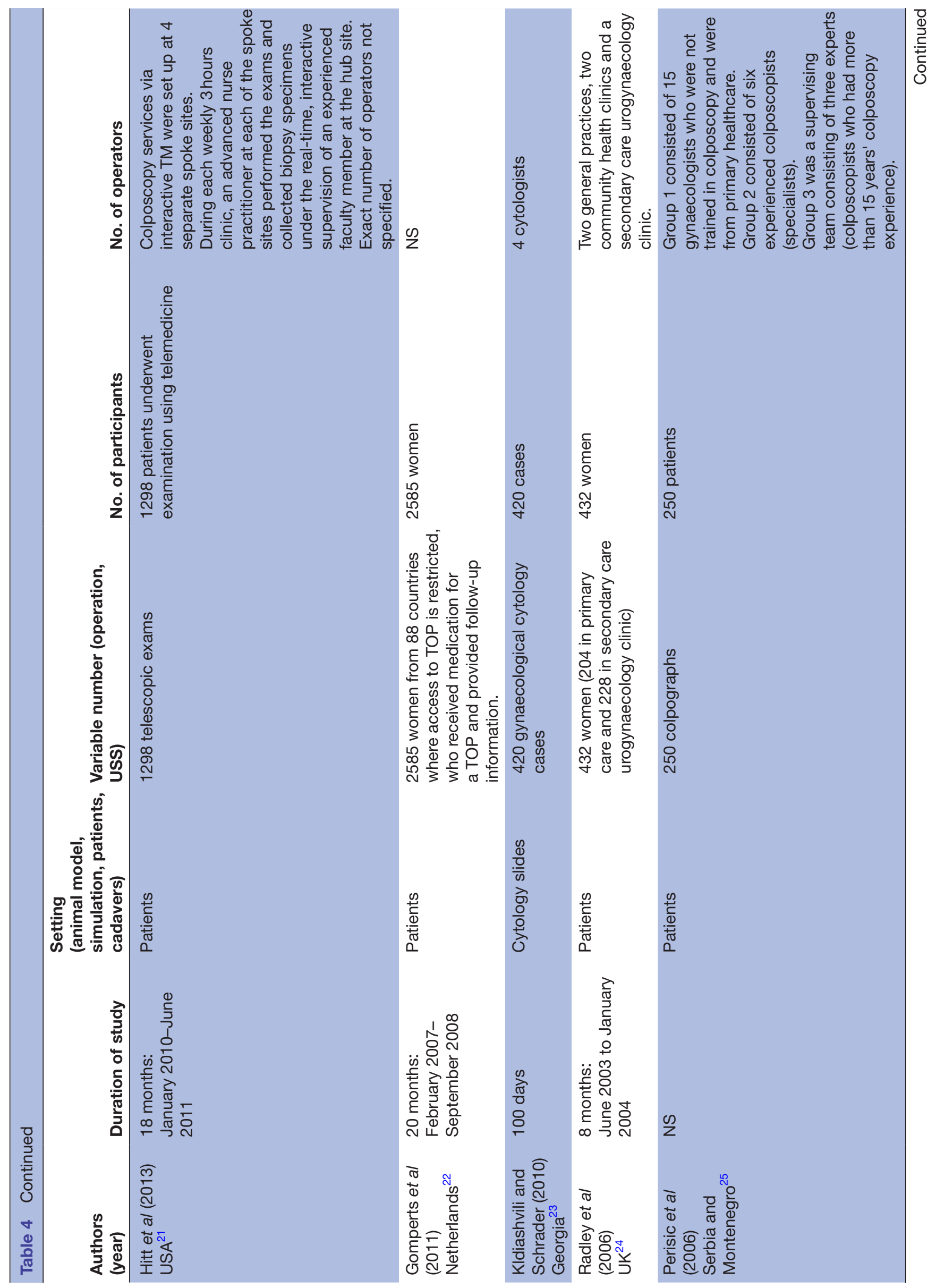


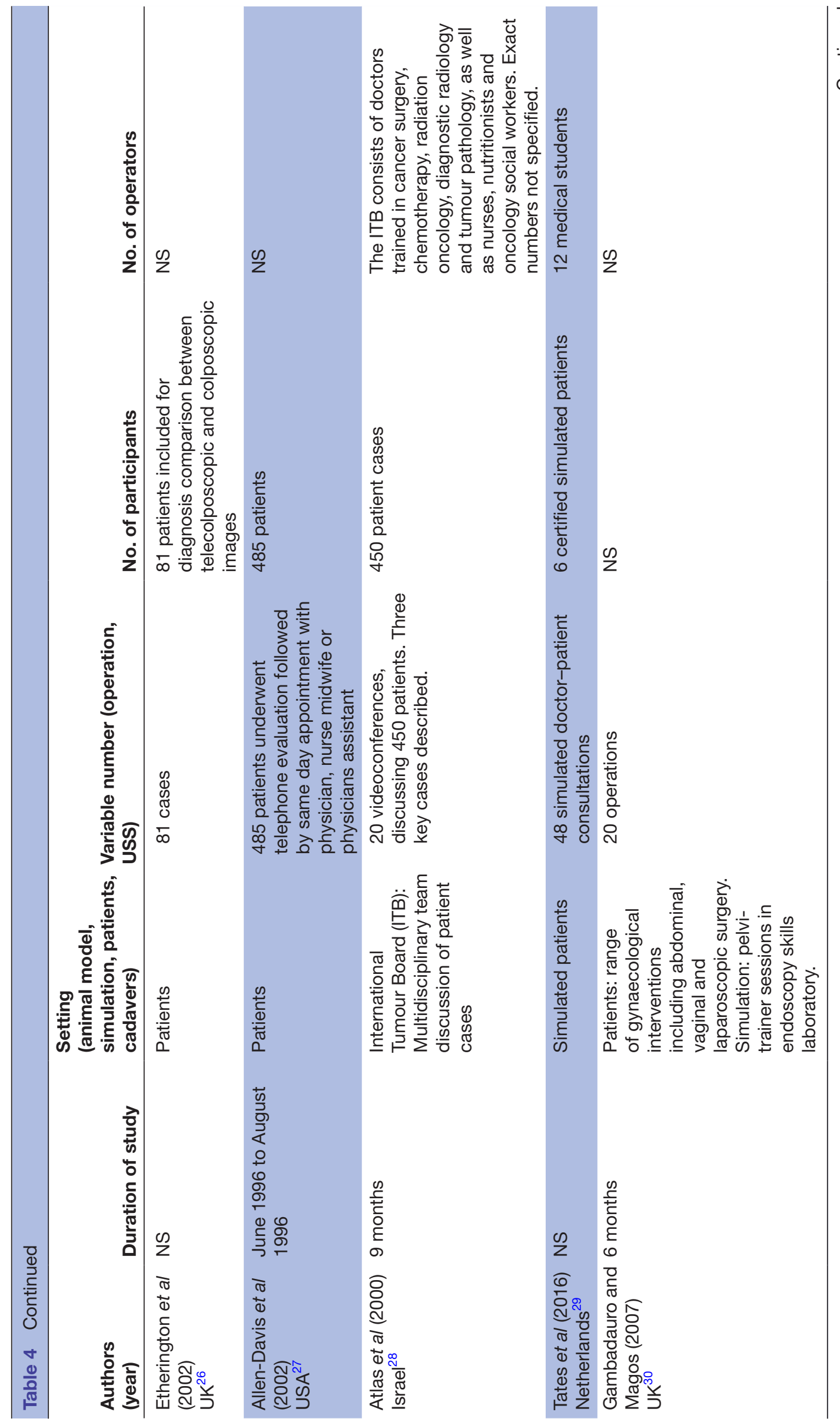



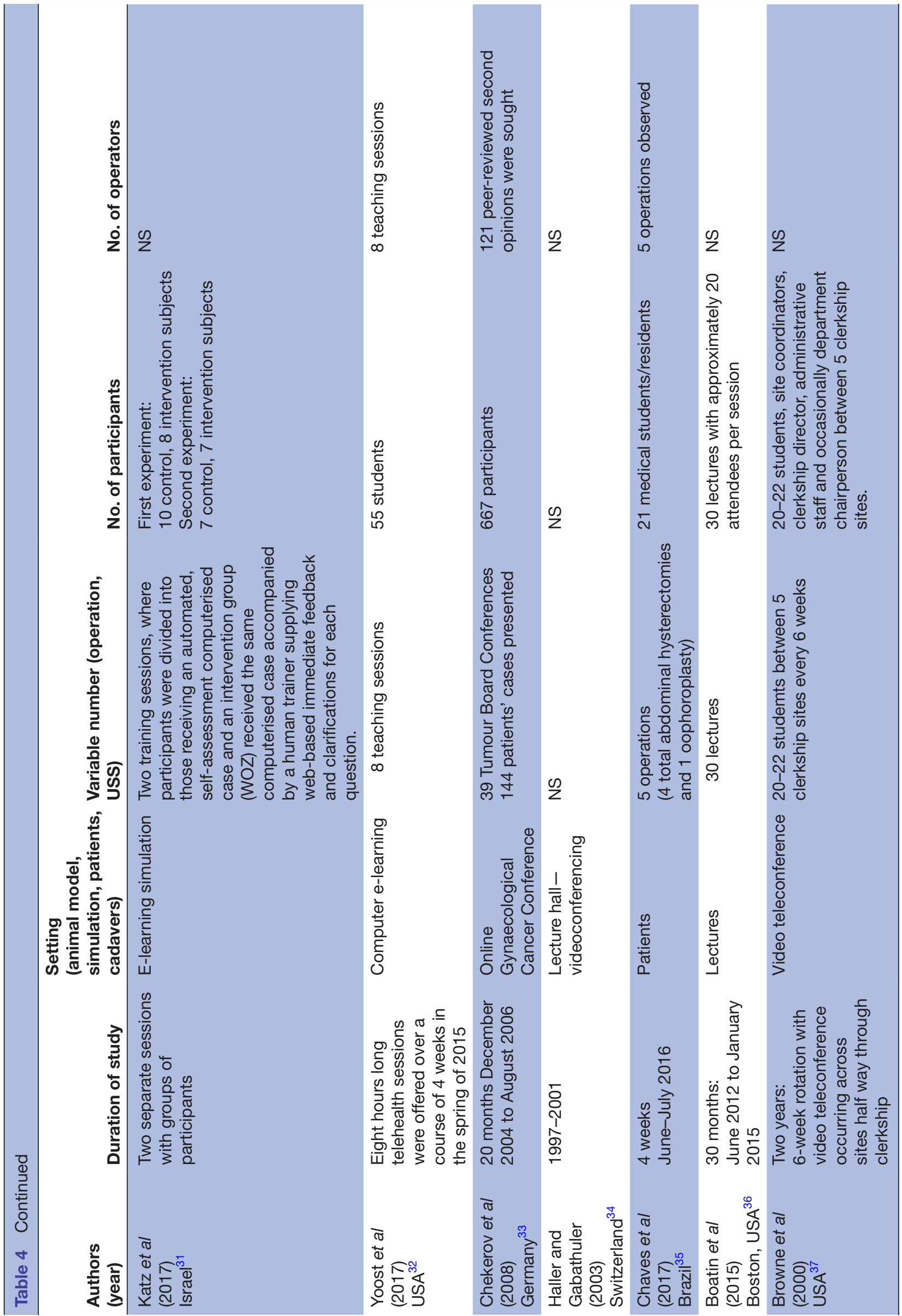


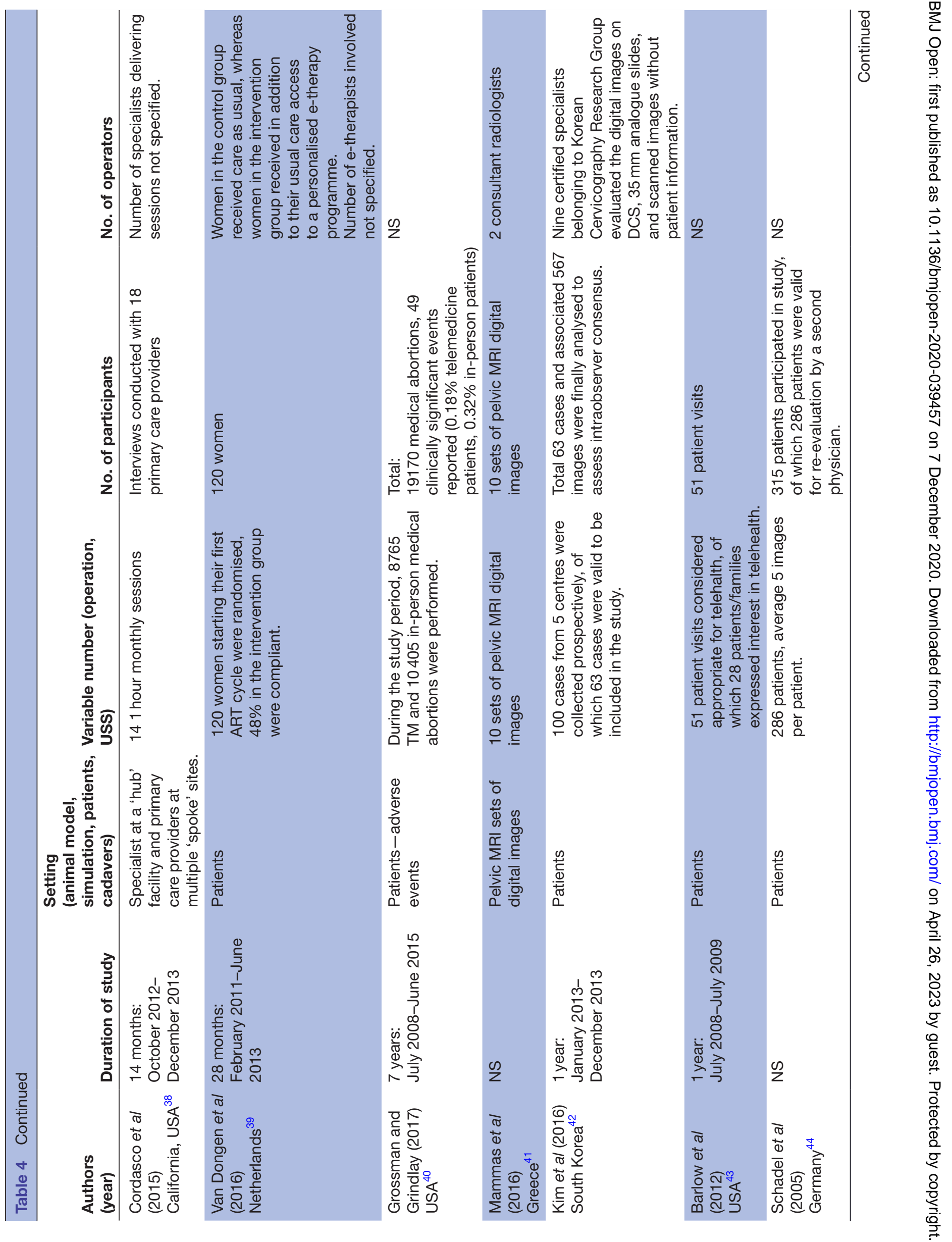




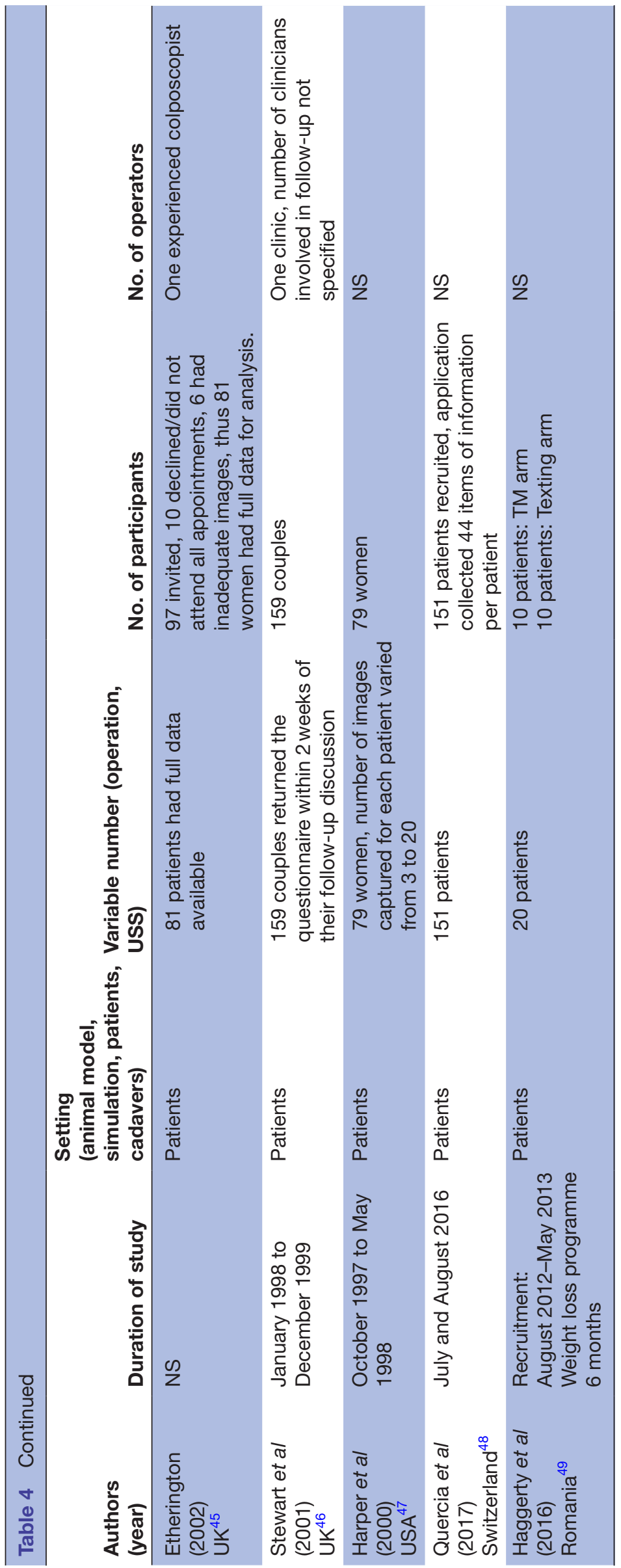

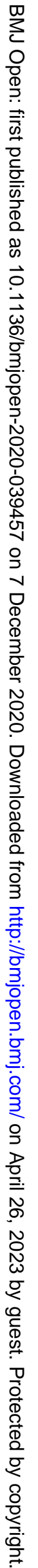


1992 to 2018. Following the initial screen, 243 studies were excluded due to the title alone and 70 abstracts were retained and examined. Of those, 15 were excluded because they were not relevant to the research questions (ie, not focused on TM), focused on other specialties, were not available in English or were duplicate studies. By duplicate studies, we refer to the retrieval of the same study through different the databases. Of the remaining 55 full-text publications that were examined, 16 were review or opinion articles and one focused on antenatal care. They were therefore excluded, leaving 39 studies for inclusion in the review. ${ }^{11-49}$ An overview of the search results and screening process is summarised in the study flow diagram (figure 1).

\section{Study characteristics}

Data from the 39 studies included in this review demonstrated considerable variation regarding the research question, methodology, study design, sample size and outcome measures. Of the 39 studies, 19 assessed gynaecological clinical practice; eight investigated gynaecological education, while one assessed the preceding pair concomitantly. 11 studies investigated the feasibility of TM within gynaecological practice. The majority of the studies were level IV evidence, as they were either case series, case-control or cohort studies. On the NewcastleOttawa quality assessment scale, 19 studies were classified as good, 12 fair and eight poor ${ }^{10}$ (table 5).

Sample sizes ranged from 6 to 19 170, although not all studies specified sample size. Of the 39 studies, the highest number were conducted in the USA $(n=13)$, followed by the Netherlands $(n=6)$, UK $(n=6)$, Switzerland $(n=4)$, Israel $(n=2)$, Germany $(n=2)$, Romania $(n=1)$, Canada $(n=1)$ Serbia and Montenegro $(n=1)$, Brazil $(n=1)$, Greece $(\mathrm{n}=1)$ and South Korea $(\mathrm{n}=1)$. Therefore, the majority originated from Europe and North America. One study was from Brazil published in 2017, ${ }^{34}$ one from South Korea published in 2016, ${ }^{41}$ one from Georgia published in $2010^{22}$ and two from Israel published in 2000 and $2017 .{ }^{2730}$ No obvious association is observed between the year of publication and the geographical region.

Various subspecialties of O\&G were investigated the most common being cervical pathology/cytology in 13 studies $^{14} 16-18212325264244454748$ followed by benign gynaecology in five studies, ${ }^{11} 19303543$ early pregnancy and medical abortion in four studies, ${ }^{12} 202240$ fertility and reproductive medicine in three studies, ${ }^{32} 3946$ urogynaecology in three studies ${ }^{152429}$ and gynaecological oncology in three studies. ${ }^{283} 49$

Different modes of TM have been used and reported in the different studies ranging from interactive and personalised web-based programmes giving preoperative and postoperative advice, ${ }^{11} 1939$ video teleconferences,,${ }^{1240}$ online/internet consultations ${ }^{20}{ }^{22}$ and telephone consultations. ${ }^{15272943464849}$ Moreover, 11 studies investigated the effectiveness of telecolposcopy where an experienced clinician had made decisions and diagnosis remotely. ${ }^{14161721252642444547} \mathrm{TM}$ has also been used for collaboration and training between clinicians and medical students; for example, e-consultations between primary and secondary care clinicians discussing the care of patients ${ }^{23}$ and in teleconferences providing expert and multidisciplinary input in oncological cases ${ }^{283341}$ Furthermore, the role of TM in medical education has been assessed in three studies where trainees were provided feedback from an experienced surgeon remotely, during real-time surgery. ${ }^{30}{ }^{31} 35$ Studies on clinical outcomes did not report any adverse effects to the patients.

\section{Colposcopy and cervical cytology}

Hitt $e t a l^{14}$ demonstrated the feasibility of telecolposcopy in 940 patients from rural areas. The assessment was done effectively by the hub-site clinician and the authors estimated mean transportation costs per patient saved to be S $\$ 33.25 .{ }^{14}$ Another study evaluated the feasibility of using smartphone digital images for the detection of Cervical Intraepithelial Neoplasia (CIN2)+in 87 HPV-positive cases. ${ }^{16}$ There was no statistical difference in the sensitivity and specificity of the detection of CIN2+ between on-site and off-site physicians. Moreover, $95.6 \%$ of all images were deemed to be very good or acceptable for diagnostic purposes. ${ }^{16}$ Similarly, Catarino et al ${ }^{17}$ involving 332 patients demonstrated that off-site diagnosis of CIN was at least similar to that reached on-site. ${ }^{17}$

Hitt $e$ t $a l^{21}$ performed 1298 telecolposcopies whereby a nurse practitioner who has been trained in the mechanics of colposcopy performed the examination under realtime interactive supervision of an experienced colposcopist at the hub site. ${ }^{22}$ The study model of using one physician with four nurse practitioner examiners and four assistants produced an hourly cost of US $\$ 321.00$, or a cost of US $\$ 40$ per exam. This compared with a traditional model incorporating four doctor examiners with four assistants producing an hourly cost of US $\$ 416.00$ or a cost per exam of US $\$ 52 .{ }^{21}$ The authors thus concluded that this method was cost effective and well received by patients.

Kldiashvili et $a t^{23}$ evaluated 'telecytology' whereby 420 cytological images were assessed by on-site and offsite cytologists. ${ }^{23}$ The study found a $94 \%$ concordance between routine versus digital images and $97 \%$ of digital images were rated as 'good' or 'excellent'. ${ }^{23}$ Another study by Etherington et al on telecolposcopy demonstrated a high sensitivity and specificity of $89 \%$ and $93 \%$ respectively on the basis of diagnosis of 'normal' and 'abnormal'. ${ }^{24}$ The images produced were of sufficient quality for diagnosis. ${ }^{26}$ Harper $e t a t^{77}$ performed coloposcopies in 79 women whereby the images were sent to a hub site for further assessment and comparison. ${ }^{47}$ The interobserver agreement was as high as $86 \%(\mathrm{k}=0.68)$ and the colposcopists' and patients' satisfaction was rated as 'excellent'. The study concluded that the telecolposcopic system is technically feasibile and is preferred by women as it cuts down travel costs. ${ }^{47}$

Perisic et al performed videocolpocopy in 250 patients. ${ }^{25}$ The diagnostic accuracy of three different groups was 
Open access

Table 5 Newcastle-Ottawa quality assessment table

\section{Authors}

(year)

Selection

Comparability

Outcome Quality

Clinical

Bouwsma et al (2018)

**** *

Netherlands ${ }^{11}$

\section{Grindlay and Grossman (2017) \\ USA $^{12}$}

Shehata et al (2016)

***

Canada $^{13}$

Hitt et al

(2016)

USA $^{14}$

Jefferis et al (2016)

$\mathrm{UK}^{15}$

Ricard-Gauthier et al (2015)

Switzerland $^{16}$

Catarino et al (2015)

Switzerland ${ }^{17}$

Stratton et al (2015)

USA $^{18}$

Noordegraaf et al (2014)

Netherlands ${ }^{19}$

Gomperts et al (2014)

Netherlands ${ }^{20}$

Hitt et al (2013)

USA $^{21}$

Gomperts et al (2011)

Netherlands ${ }^{22}$

Kldiashvili, and Schrader (2010)

Georgia $^{23}$

Radley et al (2006)

$\mathrm{UK}^{24}$

Perisic et al (2006)

Serbia and Montenegro ${ }^{25}$

Etherington et al (2002)

$\mathrm{UK}^{26}$

Allen-Davis et al (2002)

USA $^{27}$

\begin{tabular}{|c|c|c|c|c|}
\hline $\begin{array}{l}\text { Atlas et al (2000) } \\
\text { Israel }^{28}\end{array}$ & * & & $\star *$. & Poor \\
\hline $\begin{array}{l}\text { Tates et al (2016) } \\
\text { Netherlands }\end{array}$ & $* \star *$ & * & $\star \star$ & Good \\
\hline \multicolumn{5}{|l|}{ Education } \\
\hline $\begin{array}{l}\text { Gambadauro and Magos (2007) } \\
\text { UK }^{30}\end{array}$ & * & & **. & Poor \\
\hline $\begin{array}{l}\text { Yoost et al } \\
(2017) \\
\text { USA }^{32}\end{array}$ & ** & * & $\star *$ & Fair \\
\hline
\end{tabular}

Continued 
Table 5 Continued

\begin{tabular}{|c|c|c|c|c|}
\hline $\begin{array}{l}\text { Authors } \\
\text { (year) }\end{array}$ & Selection & Comparability & Outcome & Quality \\
\hline $\begin{array}{l}\text { Chekerov et al } \\
\text { (2008) } \\
\text { Germany }^{33}\end{array}$ & * & & **. & Poor \\
\hline $\begin{array}{l}\text { Haller and Gabathuler } \\
(2003) \\
\text { Switzerland }^{34}\end{array}$ & * & & $* *$ & Poor \\
\hline $\begin{array}{l}\text { Boatin et al } \\
(2015) \\
\text { Boston, USA }{ }^{36}\end{array}$ & ** & * & ** & Fair \\
\hline \multicolumn{5}{|l|}{ Clinical and education } \\
\hline $\begin{array}{l}\text { Cordasco et al } \\
(2015) \\
\text { USA }^{38}\end{array}$ & ** & * & ** & Fair \\
\hline \multicolumn{5}{|l|}{ Feasibility } \\
\hline $\begin{array}{l}\text { Van Dongen et al (2016) } \\
\text { Netherlands }\end{array}$ & $* * *$ & * & ** & Good \\
\hline $\begin{array}{l}\text { Grossman and Grindlay (2017) } \\
\text { USA }^{40}\end{array}$ & $\star \star *$ & * & $\star \star$ & Good \\
\hline $\begin{array}{l}\text { Mammas et al (2016) } \\
\text { Greece }^{41}\end{array}$ & * & & * & Poor \\
\hline $\begin{array}{l}\text { Etherington (2002) } \\
\text { UK }^{45}\end{array}$ & *** & * & ** & Good \\
\hline $\begin{array}{l}\text { Stewart et al (2001) } \\
\text { UK }^{46}\end{array}$ & $* \star *$ & * & $\star \star$ & Good \\
\hline $\begin{array}{l}\text { Harper et al (2000) } \\
\text { USA }^{47}\end{array}$ & *** & * & ** & Good \\
\hline $\begin{array}{l}\text { Quercia et al (2017) } \\
\text { Switzerland }^{48}\end{array}$ & $\star \star *$ & * & $\star \star$ & Good \\
\hline $\begin{array}{l}\text { Haggerty et al (2016) } \\
\text { Romania }^{49}\end{array}$ & *** & * & ** & Good \\
\hline
\end{tabular}

assessed (inexperienced, experienced and expert colposcopists) which showed high interobserver agreement. The authors concluded that videocolposcopy can provide an accurate diagnosis, reduced travel distance and can enable training of healthcare staff. ${ }^{25}$ Schadel $e t a l^{4}$ evaluated the use of digital colposcopy against conventional binocular colposcopy. ${ }^{44}$ Three hundred and fifteen patients had a colposcopic assessment onsite and had their images saved which were reviewed by another colposcopist. There was agreement between the examiners in $69 \%$ of cases $(\mathrm{k}=0.60)$. The authors concluded that digital colposcopy was reliable and provided advantages in terms of a better follow-up examination and internal quality control of the diagnosis. ${ }^{44}$

\section{Abortion care}

An Alaskan study evaluated the feasibility of a TM abortion care service. ${ }^{12}$ Eight providers had in-depth 
interviews about the quality of the service, which involved access to patient notes, scan images and patient video teleconference. The healthcare providers felt that the patients were assessed sooner and that the service was feasible. ${ }^{12}$ Gomperts $e t a l^{20}$ evaluated the outcome of selfadministered mifepristone and misoprostol for medical abortion in 602 Brazilian women. ${ }^{20}$ The surgical intervention rate after medical abortion was $19 \%, 15.5 \%$ and $45 \%$ at $9,10-12$ and 13 weeks gestation, respectively. The study concluded that home use of the above medication in women before 13 weeks gestation through a TM service is safe and effective. ${ }^{20}$ The same group performed a study involving 2323 women from 88 different countries to analyse the factors influencing surgical intervention after medical abortion. ${ }^{22}$ These women were assessed by TM with interactive online consultation and follow-up. There were regional differences in the rates of surgical intervention, which may reflect different clinical practice and local guidelines. ${ }^{22}$

Grossman et $a t^{40}$ compared outcomes between TM and in person management of medical abortion. This was a large study with 8765 women who had abortion management via TM and 10405 women who had in person management. The complication rates were low at $0.18 \%$ and $0.32 \%$, respectively, and this difference was non-significant. ${ }^{40}$

\section{Gynaecological surgery}

Bouwsma et $a l^{11}$ performed a randomised-controlled trial where 227 women underwent an internet-based personalised care programme after gynaecological surgery and 206 women had the usual postoperative care. ${ }^{11}$ The study demonstrated an accelerated recovery and reduced time to return to work in the intervention group which were both statistically significant. ${ }^{11}$ Moreover, the authors reported an estimated 54 euros savings per patient with the intervention group. ${ }^{11} \mathrm{~A}$ similar study by the same group recruited 110 patients who were randomly allocated to an eHealth intervention with tailored preoperative and postoperative instructions with regards to resumption of work and daily activities. ${ }^{19}$ Compared with the control group, the eHealth intervention was associated with reduced time to return to work $(\mathrm{p}=0.048)$. Furthermore, at 26 weeks post operation, the intervention group reported improved quality of life score compared with the control group $(\mathrm{p}=0.024)$.

Gambadauro et al (2007) developed a networkenhanced surgical training (NEST) telementoring system consisting of audio-visual interaction of the trainer with the trainee during gynaecological surgery. ${ }^{30}$ The authors concluded that NEST was a reliable and affordable learning tool. ${ }^{30}$ Chaves $e t a l^{35}$ evaluated the effectiveness of a telementoring programme whereby gynaecology residents and medical students received education on their smartphones connected to a wireless network though live surgery. ${ }^{35} 94 \%$ reported that the streaming video system was an effective educational tool. ${ }^{35}$

\section{DISCUSSION}

This systematic review is an analysis of where we stand with the use of TM at present; the COVID-19 pandemic has provided a catalyst for change, as it has forced us all to consider methods of remote care. The incorporation of TM into daily practice became a priority to help limit the spread of disease by limiting face-to-face contact. Our review presents the evidence of the promising role of TM within gynaecological practice and education. Of note, the majority of the selected studies were classified as good or fair using the Newcastle-Ottawa methodological assessment tool. The applications of TM to date have been shown to be safe and effective in providing remote care and training. This is in line with a recent review conducted in the field of general surgery. ${ }^{50}$

\section{Clinical potential}

Particular fields within gynaecology inherently lend themselves to TM approaches. This is evident by the number of studies focused on particular areas; of the 22 clinically focused studies, eight were within colposcopy $^{1315-1720222425}$ with seven of them being classified as good and fair methodological quality. Moreover, the studies analysed in this review clearly demonstrate that telementoring can improve the education of medical professionals within gynaecology and may occupy a niche in surgical education by enabling the education of surgeons within and between hospitals. It has been shown in some settings to be a safe and effective method of implementing remote mentoring. Furthermore, Boatin et $a l^{36}$, a study of fair methodological quality, demonstrated that successful video conferencing can result in expertise being shared bilaterally and internationally across individuals who potentially are unable to travel. It could therefore be beneficial to both resource-rich and resource-poor institutions. ${ }^{36}$

The most consistent finding was that digital colposcopy was reliable and provided advantages with a better follow-up examination and quality control of the diagnosis. The cost varied depending on the particular use of TM and only a few studies explicitly outlined the cost of implementation of their TM systems. The initial cost must be weighed against potential saving such as reduced travel time, improved access to expert healthcare and thus potentially earlier diagnosis, and the long-term benefit of increased healthcare expertise via a wider education audience. This is a complex cost-benefit analysis, which will vary with type and setting of TM implementation and is certainly something to be considered in future studies.

\section{Telementoring value}

Robotic surgery has been demonstrated to have a role in telementoring in general surgery. ${ }^{51}$ No studies focusing on the use of robotic surgery for telementoring in gynaecology are currently available. One study compared clinical outcomes between telementoring and on-site mentoring of robotic assisted laparoscopic radical prostatectomy found no significant difference between the 
two. ${ }^{52}$ This finding suggests there may be a further role for telementoring in gynaecological cases using robotic surgery as this technology becomes more widespread. Another emerging technology to consider is Google Glass, a wearable device that provides users hands-free access to computer functions. The potential benefit of this technology in telementoring has been demonstrated in both surgical and non-surgical medical settings. ${ }^{53} 54$ Moreover, more recent telecommunication technologies would certainly help to improve the perception of telementoring, particularly the advent of augmented and virtual reality. ${ }^{55}$ Again, there are no studies identified specific to gynaecology, however, based on the studies available from other specialties, it could well be piloted within gynaecology.

TM is unlikely to ever supersede on-site mentoring completely but should be used as an adjunct to traditional clinical practice and training. At present, there may be technical limitations to its widespread implementation owing to suboptimal internet penetration particularly in low-income countries and the fact that internet connectivity is concentrated within cities globally. ${ }^{50} \mathrm{In}$ addition, the necessary infrastructure including telecommunications, specific electrical appliances and technical expertise of the personnel including the clinicians may be suboptimal in developing countries. Furthermore, highly motivated individuals are required to implement TM programmes whether that might be for clinical or educational purposes. Moreover, when it comes to telementoring, bandwidth should be over $521 \mathrm{~kb} / \mathrm{s}$ and none of the selected studies reported such figure. ${ }^{3}$ Security will also play a crucial role in developing TM programmes in the future as this involves sharing and handling sensitive patient information. Therefore, the development of agreed frameworks to ensure that personal and sensitive information is encrypted appropriately is essential.

\section{Limitations of the current evidence}

The majority of studies included were observational in nature, without clear quantitative outcomes for statistical analysis and were classified as level IV evidence according to the Oxford Centre for Evidence-based Medicine preform. ${ }^{10}$ In a number of studies, the variable was low and thus the conclusion of the study was based on a single operator demonstrating a TM application within gynaecology. Therefore, there is the potential for a number of the included studies to be subject to selection bias, with certain patients, practitioners or students more amenable to the technology as it was more likely to be involved in the study resulting in positive outcomes with TM.

On the other hand, some studies included thousands of patients, in particular those focused on telecolposcopy and TM abortion care. These studies indicate the widereaching nature of these applications. In these cases, one must consider that often low numbers of operators were involved and thus again selection bias may apply. In the future, randomised controlled studies involving larger numbers of patients and operators with measurable outcomes are required in order to be able to draw reliable conclusions.

\section{CONCLUSION}

This review is to our knowledge the only such review on the topic. The range of studies demonstrates the wide potential role of TM within gynaecology. However, this variety translates to broad conclusions based mostly on self-reported data. Reassuringly, the methodological quality of the majority of the reviewed studies were classified as good or fair. While some promising studies have been conducted, there is yet insufficient evidence to support the clinical or cost effectiveness of TM in gynaecology. The role of TM has more than ever become more obvious as we are undergoing the COVID-19 pandemic where effective TM strategies need to be implemented to provide safe and effective care to patients, without putting patients, doctors and the community at risk of the infection.

Contributors SM wrote the manuscript. SM and SS were involved in the study design. SM and NG were involved in the literature search, analysis of studies and drafting of the manuscript. BPJ, MC, TB-M, YA-S and KG were involved in data collection. DT, JY, TB and SS were responsible for supervision or mentorship. SS is the senior author of the manuscript, generated the topic of the manuscript and takes responsibility for its content. Each author contributed important intellectual content during manuscript drafting or revision and accepts accountability for the overall work by ensuring that questions pertaining to the accuracy or integrity of any portion of the work are appropriately investigated and resolved. SS Senior Author.

Funding The authors have not declared a specific grant for this research from any funding agency in the public, commercial or not-for-profit sectors.

\section{Competing interests None declared.}

Patient and public involvement Patients and/or the public were not involved in the design, or conduct, or reporting, or dissemination plans of this research.

Patient consent for publication Not required.

Provenance and peer review Not commissioned; externally peer reviewed.

Data availability statement Data are available in a public, open access repository. Systematic Review.

Open access This is an open access article distributed in accordance with the Creative Commons Attribution Non Commercial (CC BY-NC 4.0) license, which permits others to distribute, remix, adapt, build upon this work non-commercially, and license their derivative works on different terms, provided the original work is properly cited, appropriate credit is given, any changes made indicated, and the use is non-commercial. See: http://creativecommons.org/licenses/by-nc/4.0/.

\section{ORCID iDs}

Sughashini Murugesu http://orcid.org/0000-0001-8852-0892

Benjamin P Jones http://orcid.org/0000-0002-0391-0443

\section{REFERENCES}

1 Becker S, Miron-Shatz T, Schumacher N, et al. mHealth 2.0: experiences, possibilities, and perspectives. JMIR Mhealth Uhealth 2014;2:e24.

2 World Health Organization. Telemedicine: opportunities and developments in member states: report on the second global survey on eHealth. World Health organization. ISBN: 978-92-4-1564144, 2010. Available: http://www.who.int/goe/publications/goe telemedicine_2010.pdf

3 Schlachta CM, Nguyen NT, Ponsky T, et al. Project 6 Summit: SAGES telementoring initiative. Surg Endosc 2016;30:3665-72. 
4 Singh GK, Miller BA, Hankey BF, et al. Persistent area socioeconomic disparities in U.S. incidence of cervical cancer, mortality, stage, and survival, 1975-2000. Cancer 2004;101:1051-7.

5 Coughlin SS, Leadbetter S, Richards T, et al. Contextual analysis of breast and cervical cancer screening and factors associated with health care access among United States women, 2002. Soc Sci Med 2008:66:260-75.

6 Lindau ST, Tomori C, Lyons T, et al. The association of health literacy with cervical cancer prevention knowledge and health behaviors in a multiethnic cohort of women. Am J Obstet Gynecol 2002;186:938-43.

7 Birkmeyer JD, Siewers AE, Finlayson EVA, et al. Hospital volume and surgical mortality in the United States. $N$ Engl $J$ Med 2002;346:1128-37.

8 Moher D, Liberati A, Tetzlaff J, et al. Reprint--preferred reporting items for systematic reviews and meta-analyses: the PRISMA statement. Phys Ther 2009;89:873-80.

9 Theodosiou T, Vizirianakis IS, Angelis L, et al. MeSHy: mining unanticipated PubMed information using frequencies of occurrences and concurrences of mesh terms. J Biomed Inform 2011;44:919-26.

10 Oxford centre for Evidenced-based Medicine- levels of evidence. Available: https://www.cebm.net/2009/06/oxford-centre-evidencebased-medicine-levels-evidence-march-2009/ [Accessed July 2019].

11 Bouwsma EVA, Huirne JAF, van de Ven PM, et al. Effectiveness of an Internet-based perioperative care programme to enhance postoperative recovery in gynaecological patients: cluster controlled trial with randomised stepped-wedge implementation. BMJ Open 2018;8:e017781:30.

12 Grindlay K, Grossman D. Telemedicine provision of medical abortion in Alaska: through the provider's lens. J Telemed Telecare 2017:23:680-5

13 Shehata F, Posner G, Afkham A, et al. Evaluation of an electronic consultation service in obstetrics and gynecology in Ontario. Obstet Gynecol 2016;127:1033-8.

14 Hitt WC, Low GM, Lynch CE, et al. Application of a Telecolposcopy program in rural settings. Telemed J E Health 2016;22:816-20.

15 Jefferis $\mathrm{H}$, Muriithi $\mathrm{F}$, White B, et al. Telephone follow-up after day case tension-free vaginal tape insertion. Int Urogynecol J 2016;27:787-90.

16 Ricard-Gauthier D, Wisniak A, Catarino R, et al. Use of Smartphones as adjuvant tools for cervical cancer screening in low-resource settings. J Low Genit Tract Dis 2015;19:295-300.

17 Catarino R, Vassilakos P, Scaringella S, et al. Smartphone use for cervical cancer screening in low-resource countries: a pilot study conducted in Madagascar. PLoS One 2015;10:e0134309:29.

18 Stratton SL, Spencer HJ, Greenfield WW, et al. A novel use of a statewide telecolposcopy network for recruitment of participants in a phase I clinical trial of a human papillomavirus therapeutic vaccine. Clin Trials 2015;12:199-204.

19 Vonk Noordegraaf A, Anema JR, van Mechelen W, et al. A personalised eHealth programme reduces the duration until return to work after gynaecological surgery: results of a multicentre randomised trial. BJOG 2014;121:1127-36.

20 Gomperts R, van der Vleuten K, Jelinska K, et al. Provision of medical abortion using telemedicine in Brazil. Contraception 2014;89:129-33.

21 Hitt WC, Low G, Bird TM, et al. Telemedical cervical cancer screening to bridge Medicaid service care gap for rural women. Telemed $J E$ Health 2013;19:403-8.

22 Gomperts R, Petow SAM, Jelinska K, et al. Regional differences in surgical intervention following medical termination of pregnancy provided by telemedicine. Acta Obstet Gynecol Scand 2012;91:226-31.

23 KIdiashvili E, Schrader T. Diagnostic accuracy and image quality using a USB digital eyepiece camera for telecytology-Georgian experience. Telemed J E Health 2010;16:1051-2.

24 Radley SC, Jones GL, Tanguy EA, et al. Computer interviewing in urogynaecology: concept, development and psychometric testing of an electronic pelvic floor assessment questionnaire in primary and secondary care. BJOG 2006;113:231-8.

25 Perisic Z, Rasic R, Raznatovic S. Quality and efficacy of a telecolposcopy programme. J Telemed Telecare 2005;11:20-2.

26 IJ E, Watts AD, Hughes E, et al. The use of telemedicine in primary care for women with cervical cytological abnormalities. $J$ Telemed Telecare 2002;8:17-19.

27 Allen-Davis JT, Beck A, Parker R, et al. Assessment of vulvovaginal complaints: accuracy of telephone triage and in-office diagnosis. Obstet Gynecol 2002;99:18-22.

28 Atlas I, Granai CO, Gajewski W, et al. Videoconferencing for gynaecological cancer care: an international tumour board. $J$ Telemed Telecare 2000;6:242-4.
29 Tates K, Antheunis ML, Kanters S, et al. The effect of Screento-Screen versus face-to-face consultation on doctor-patient communication: an experimental study with simulated patients. $J$ Med Internet Res 2017;19:e421:20.

30 Gambadauro P, Magos A. Nest (network enhanced surgical training): a PC-based system for telementoring in gynaecological surgery. Eur J Obstet Gynecol Reprod Biol 2008;139:222-5.

31 Katz A, Tepper R, Shtub A. Simulation Training: Evaluating the Instructor's Contribution to a Wizard of Oz Simulator in Obstetrics and Gynecology Ultrasound Training. JMIR Med Educ 2017;3:e8:21.

32 Yoost JL, Starcher RW, King-Mallory RA, et al. The use of telehealth to teach reproductive health to female rural high school students. $J$ Pediatr Adolesc Gynecol 2017;30:193-8.

33 Chekerov R, Denkert C, Boehmer D, et al. Online tumor conference in the clinical management of gynecological cancer: experience from a pilot study in Germany. Int J Gynecol Cancer 2008;18:1-7.

34 Haller U, Gabathuler H. Telemedical training at the Department of gynaecology, university hospital Zürich. Curr Probl Dermatol 2003;32:39-42.

35 Chaves RO, de Oliveira PAV, Rocha LC, et al. An innovative streaming video system with a point-of-view head camera transmission of surgeries to Smartphones and tablets: an educational utility. Surg Innov 2017;24:462-70.

36 Boatin A, Ngonzi J, Bradford L, et al. Teaching by Teleconference: a model for distance medical education across two continents. Open $J$ Obstet Gynecol 2015;5:754-61.

37 Browne MW, Hines JF, Satin AJ, et al. Videoteleconferencing for administration of a multisite obstetrics and gynecology core clerkship. Obstet Gynecol 2000;95:461-3.

38 Cordasco KM, Zuchowski JL, Hamilton AB, et al. Early lessons learned in implementing a women's health educational and virtual consultation program in Va. Med Care 2015;53:S88-92.

39 van Dongen AJCM, Nelen WLDM, IntHout J, et al. e-Therapy to reduce emotional distress in women undergoing assisted reproductive technology (art): a feasibility randomized controlled trial. Hum Reprod 2016;31:1046-57.

40 Grossman D, Grindlay K. Safety of medical abortion provided through telemedicine compared with in person. Obstet Gynecol 2017; 130:778-82.

41 Mammas CS, Saatsakis G, Poulou L, et al. Telemedicine systems in uterine transplant: a feasibility and reliability study of the Pre-Grafting Teleradiological evaluation of the uterus graft. Stud Health Technol Inform 2016;226:21-4.

$42 \mathrm{Kim}$ SN, Kim YH, Nam KH, et al. Korean cervicography Research Group. development and validation of novel digitalized cervicography system. Obstet Gynecol Sci 2016;59:227-32.

43 Barlow E, Aggarwal A, Johnstone J, et al. Can paediatric and adolescent gynecological care be delivered via telehealth? Paediatr Child Health 2012;17:e12-15

44 Schädel D, Coumbos A, Ey S, et al. Evaluation of a digital store-andforward colposcopic system--a pilot study to assess usability for telemedicine. J Telemed Telecare 2005;11:103-7.

45 Etherington IJ. Telecolposcopy - a feasibility study in primary care. $J$ Telemed Telecare 2002;8:22-4.

46 Stewart L, Hamilton M, McTavish A, et al. Randomized controlled trial comparing couple satisfaction with appointment and telephone follow-up consultation after unsuccessful IVF/ICSI treatment. Hum Fertil 2001;4:249-55

47 Harper DM, Moncur MM, Harper WH, et al. The technical performance and clinical feasibility of telecolposcopy. J Fam Pract 2000;49:623-7.

48 Quercia K, Tran PL, Jinoro J, et al. A mobile health data collection system for remote areas to monitor women participating in a cervical cancer screening campaign. Telemed J E Health 2017;28.

49 Haggerty AF, Huepenbecker S, Sarwer DB, et al. The use of novel technology-based weight loss interventions for obese women with endometrial hyperplasia and cancer. Gynecol Oncol 2016;140:239-44.

50 Erridge S, Yeung DKT, Patel HRH, et al. Telementoring of surgeons: a systematic review. Surg Innov 2019;26:95-111.

51 Santomauro M, Reina GA, Stroup SP. L'Esperance JO. Telementoring in robotic surgery. Curr Opin Urol 2013;23:141-5.

52 Hinata N, Miyake H, Kurahashi T, et al. Novel telementoring system for robot-assisted radical prostatectomy: impact on the learning curve. Urology 2014;83:1088-92.

53 Wei NJ, Dougherty B, Myers A, et al. Using Google glass in surgical settings: systematic review. JMIR Mhealth Uhealth 2018;6:e54:6.

54 Dougherty B, Badawy SM. Using Google glass in nonsurgical medical settings: systematic review. JMIR Mhealth Uhealth 2017:5:e159:19. 
55 Jarc AM, Stanley AA, Clifford T, et al. Proctors exploit threedimensional ghost tools during clinical-like training scenarios: a preliminary study. World J Urol 2017;35:957-65.
56 Vera AM, Russo M, Mohsin A, et al. Augmented reality telementoring (art) platform: a randomized controlled trial to assess the efficacy of a new surgical education technology. Surg Endosc 2014;28:3467-72. 